\title{
THE MULTI-TREE CUBING ALGORITHM FOR COMPUTING ICEBERG CUBES
}

\author{
A Thesis \\ Submitted to the Faculty of Graduate Studies and Research \\ in Partial Fulfillment of the Requirements \\ for the Degree of \\ Master of Science \\ in Computer Science \\ University of Regina
}

By

Xing Li

Regina, Saskatchewan

June 2005

(C) Copyright 2005: Xing Li 


$\begin{array}{ll}\begin{array}{l}\text { Library and } \\ \text { Archives Canada }\end{array} & \begin{array}{l}\text { Bibliothèque et } \\ \text { Archives Canada }\end{array} \\ \begin{array}{l}\text { Published Heritage } \\ \text { Branch }\end{array} & \begin{array}{l}\text { Direction du } \\ \text { Patrimoine de l'édition }\end{array} \\ \begin{array}{l}\text { 395 Wellington Street } \\ \text { Ottawa ON K1A ON4 }\end{array} & \begin{array}{l}\text { 395, rue Wellington } \\ \text { Ottawa ON K1A ON4 } \\ \text { Canada }\end{array}\end{array}$

Your file Votre référence

ISBN: 0-494-06004-2

Our file Notre référence

ISBN: 0-494-06004-2

NOTICE:

The author has granted a nonexclusive license allowing Library and Archives Canada to reproduce, publish, archive, preserve, conserve, communicate to the public by telecommunication or on the Internet, loan, distribute and sell theses worldwide, for commercial or noncommercial purposes, in microform, paper, electronic and/or any other formats.

The author retains copyright ownership and moral rights in this thesis. Neither the thesis nor substantial extracts from it may be printed or otherwise reproduced without the author's permission.
AVIS:

L'auteur a accordé une licence non exclusive permettant à la Bibliothèque et Archives Canada de reproduire, publier, archiver, sauvegarder, conserver, transmettre au public par télécommunication ou par l'Internet, prêter, distribuer et vendre des thèses partout dans le monde, à des fins commerciales ou autres, sur support microforme, papier, électronique et/ou autres formats.

L'auteur conserve la propriété du droit d'auteur et des droits moraux qui protège cette thèse. $\mathrm{Ni}$ la thèse ni des extraits substantiels de celle-ci ne doivent être imprimés ou autrement reproduits sans son autorisation.
In compliance with the Canadian

Privacy Act some supporting forms may have been removed from this thesis.

While these forms may be included in the document page count, their removal does not represent any loss of content from the thesis.
Conformément à la loi canadienne sur la protection de la vie privée, quelques formulaires secondaires ont été enlevés de cette thèse.

Bien que ces formulaires aient inclus dans la pagination, il n'y aura aucun contenu manquant.

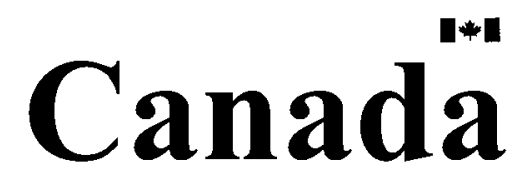




\section{UNIVERSITY OF REGINA}

\section{FACULTY OF GRADUATE STUDIES AND RESEARCH}

\section{SUPERVISORY AND EXAMINING COMMITTEE}

Xing Li, candidate for the degree of Master of Science, has presented a thesis titled, The Multi-Tree Cubing Algorithm for Computing Iceberg Cubes, in an oral examination held on April 1,2005. The following committee members have found the thesis acceptable in form and content, and that the candidate demonstrated satisfactory knowledge of the subject material.

External Examiner: Dr. Allen Herman, Department of Mathematics and Statistics

Supervisor: Dr. Howard Hamilton, Department of Computer Science

Committee Member: $\quad$ Dr. Lawrence V. Saxton, Department of Computer Science

Committee Member: $\quad$ Dr. Robert Hilderman, Department of Computer Science

Chair of Defense: Dr. Scott Murphy,

Department of Chemistry and Biochemistry 


\begin{abstract}
The computation of data cubes is one of the major operations in on-line analytical processing (OLAP), but it is also one of the most expensive. To improve efficiency, an iceberg cube represents only the cells whose aggregate value is above a given threshold (minimum support). Two existing approaches, top-down and bottom-up, are used to compute the iceberg cube for a data set, but both have performance limitations.

In this thesis, a new algorithm, called Multi-Tree Cubing (MTC), is proposed and implemented for computing an iceberg cube. Overall, the Multi-Tree Cubing algorithm is a top-down approach, so it features shared computation. By processing the orderings in the opposite order from a top-down approach called Top-Down Computation, the MTC algorithm is able to prune attributes at the partition level, which is more accurate than the pruning in a bottom-up approach called Bottom-Up Computation (BUC). The MTC algorithm is based on a specialized type of prefix tree data structure, called a AP-tree, which facilitates fast sorting and Apriori-like pruning. Once a tree is constructed, MTC traverses the tree in a depth-first manner and checks each partition against the minimum support to determine if it should output a result or prune the current branch. The results of five series of experiments comparing MTC versus BUC on real and synthetic databases are presented. The experimental results showed that MTC consistently performed the same as or better than BUC for all five series of experiments.
\end{abstract}




\section{Acknowledgements}

I would like to thank all of the people who helped me with all aspects of my research, including the preparation of this thesis. I would also like to thank the Department of Computer Science and the Faculty of Graduate Studies and Research for giving me an excellent opportunity to pursue a Master's Degree at the University of Regina. Especially, many sincere thanks go to Dr. Hamilton, who guided, helped and encouraged me each step of the way. I am very lucky and proud to be one of his students. Just as Brock Barber, one of his previous students, said in his thesis acknowledgment, "he is a rare combination of excellent researcher, supervisor, educator and most importantly, a fine human being." In addition, I would like to thank other members of the supervisory committee, Dr. Robert Hilderman and Dr. Larry Saxton, for their time and efforts. 


\section{Post Defense Acknowledgement}

I would like to thank Dr. Allen Herman for being the External Examiner of this thesis. I was impressed by his thorough reading of my thesis, and I appreciated the corrections and comments he gave me. 


\section{Dedication}

I have learned a great deal from my graduate studies and completing the M.Sc. program has become an important achievement of my life. But, what makes my life worthwhile is my family. I owe my husband Li and my son Jeffrey a lot for the time that we perhaps should have spent together. Without their support, patience, and understanding, this thesis would not have been possible. The thesis is also for my parents who inspired and encouraged me to study Computer Science when I was a young university student in China. Since then, I have always enjoyed studying and working in the field of Computer Science. 


\section{Table of Contents}

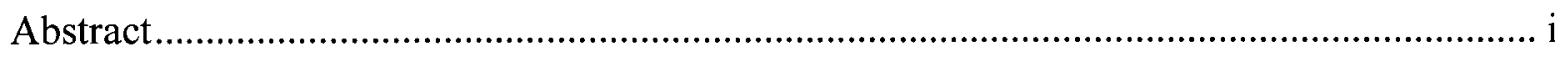

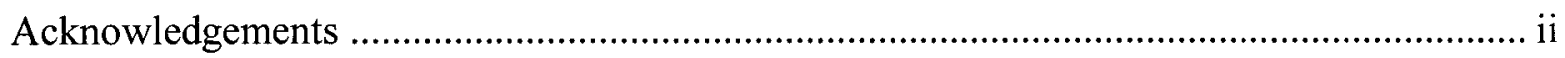

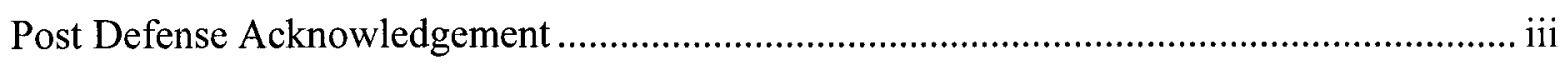

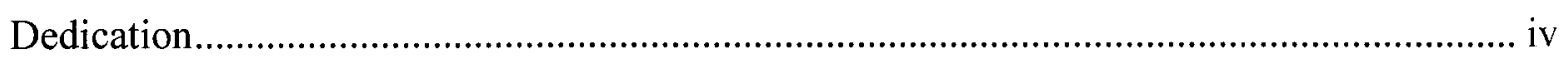

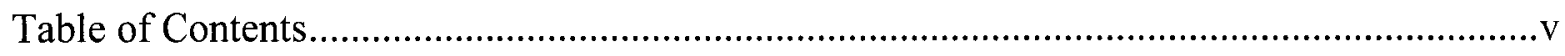

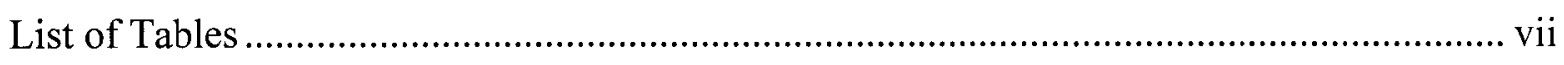

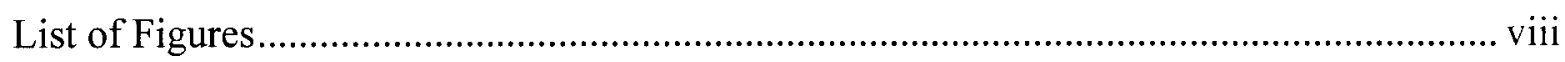

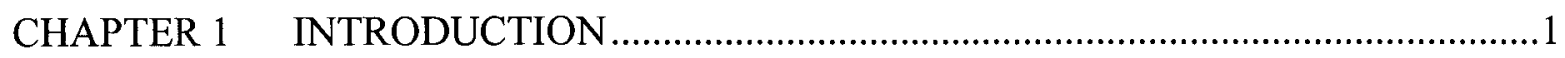

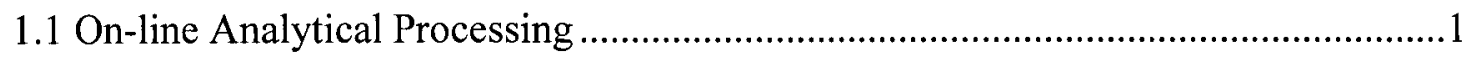

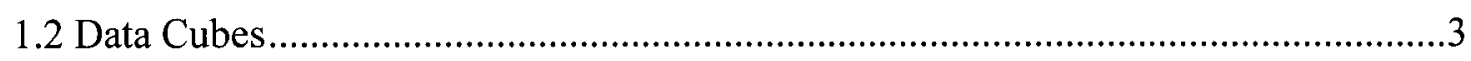

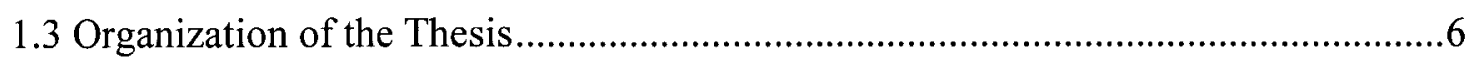

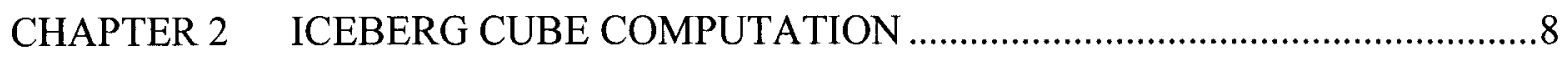

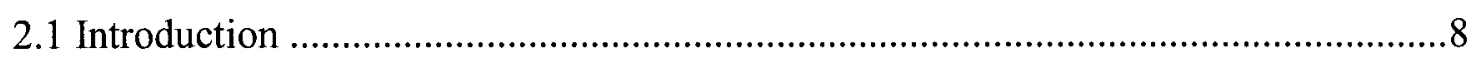

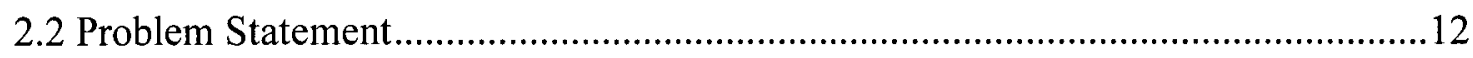

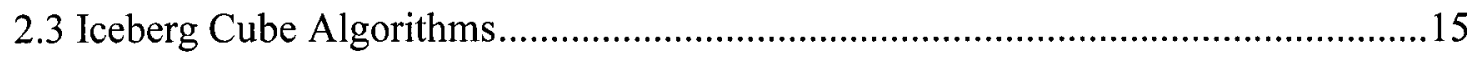

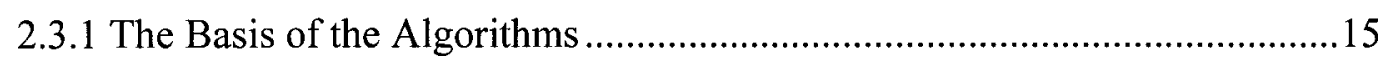

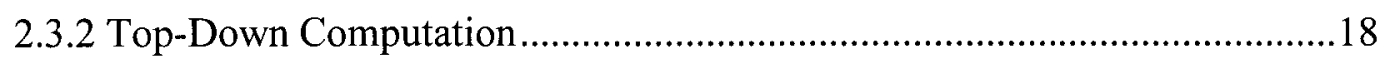

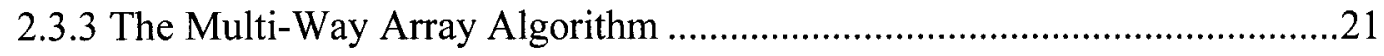

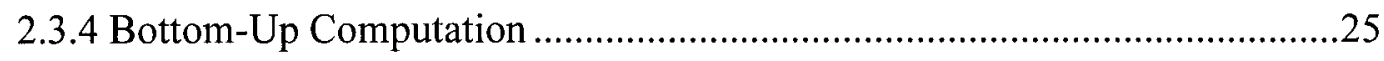

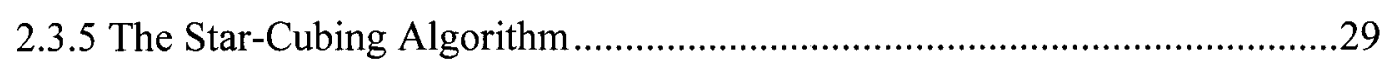

2.3.6 Algorithms for Iceberg Cubes with Complex Measures ............................32 
2.3.7 Comparison of the Iceberg Cube Algorithms

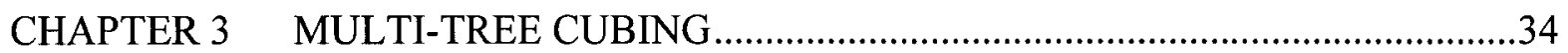

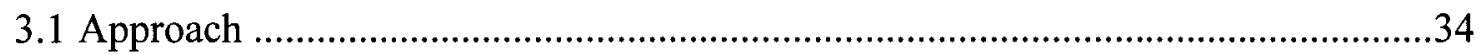

3.1.1 The Multi-Tree Cubing Algorithm ..........................................................36

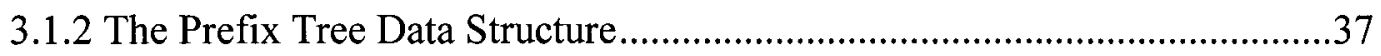

3.1.3 An Example of the Multi-Tree Cubing Algorithm ...................................39

3.2 Comparison of Multi-Tree Cubing and Other Iceberg Cube Algorithms................43

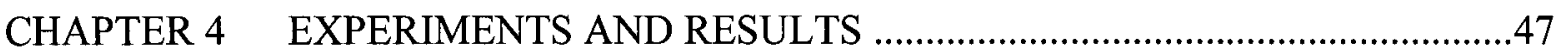

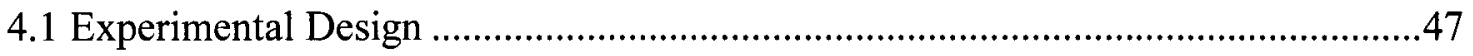

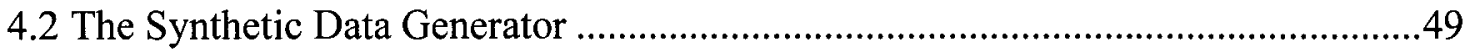

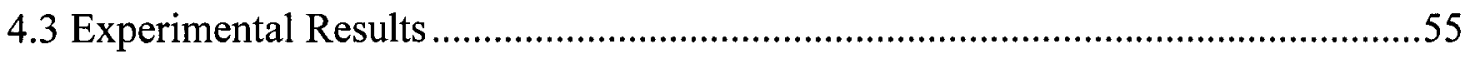

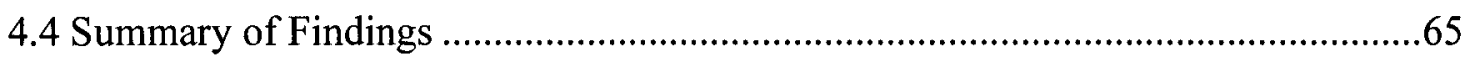

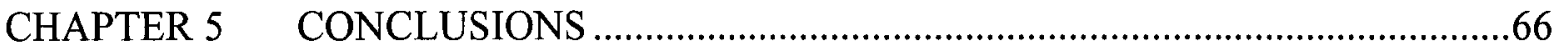

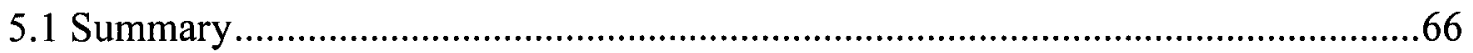

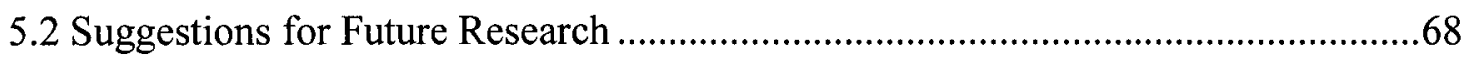

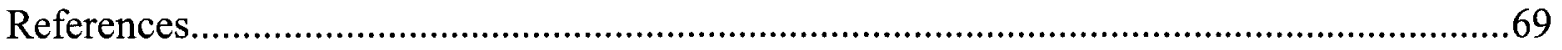

Appendix A User's Manual for Multi-Tree Cubing (MTC) and Bottom-Up

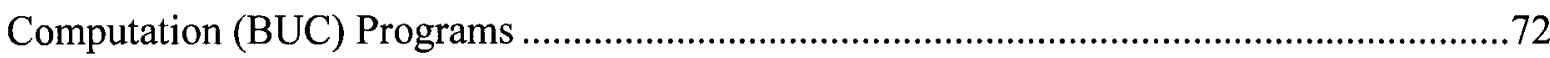




\section{List of Tables}

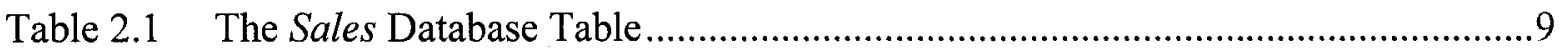

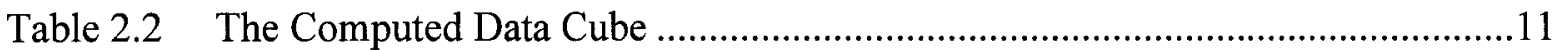

Table 2.3 Results for the Example GROUP BY Query ..............................................12

Table 2.4 The Computed Iceberg Cube (MinSup = 2) ..................................................14

Table 2.5 Summary of the TDC Computation Process .................................................20

Table 2.6 A 2-Dimenisonal Array for Four Attributes ..................................................23

Table 2.7 Summary of BUC Computation Process .....................................................28

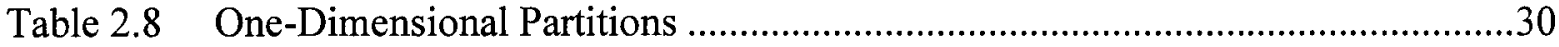

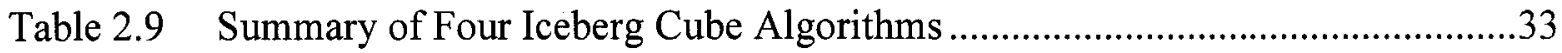

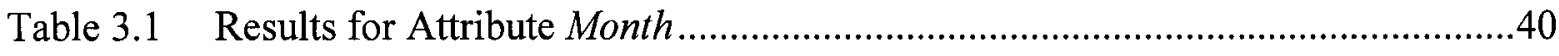

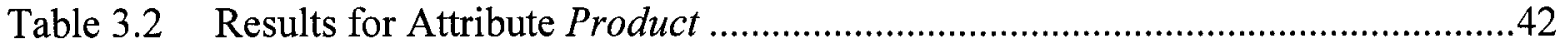

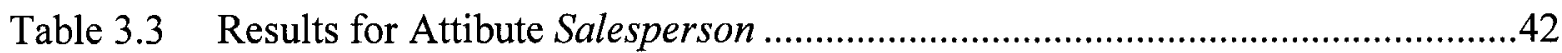

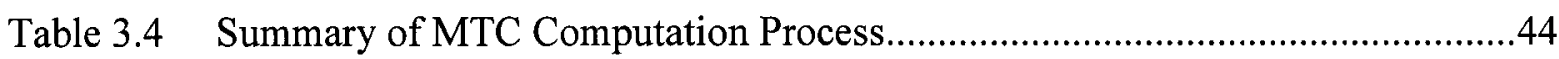

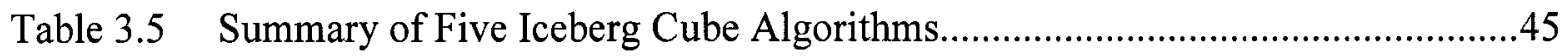

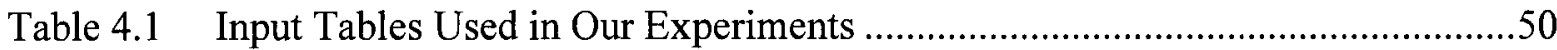




\section{List of Figures}

Figure 1.1 Specification of a Sample Iceberg Cube .......................................................5

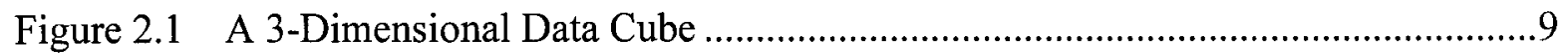

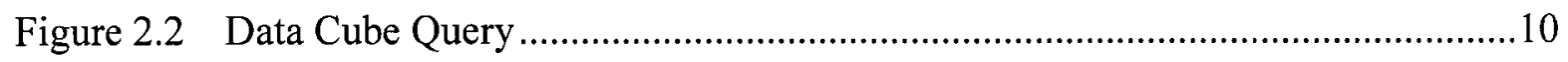

Figure 2.3 An Example of a GROUP BY Query................................................... 12

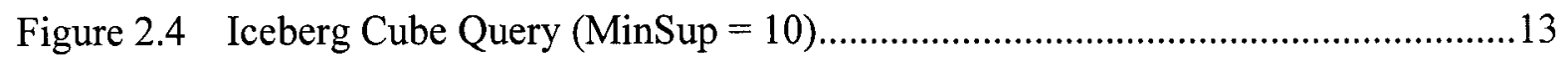

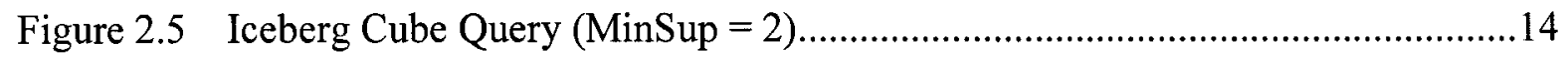

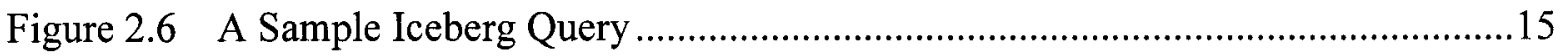

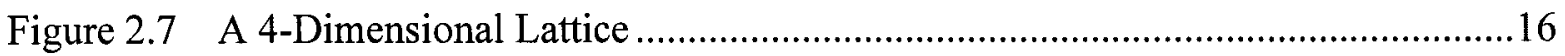

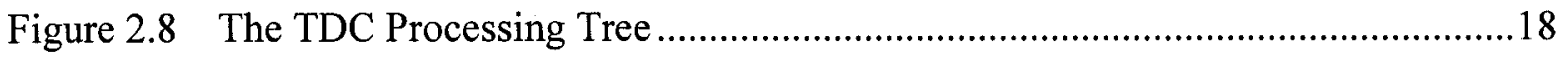

Figure 2.9 Multi-Way Array Processing Tree ...........................................................24

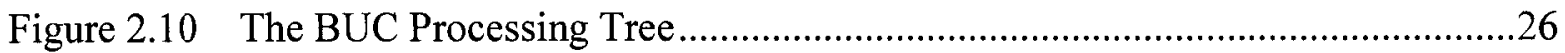

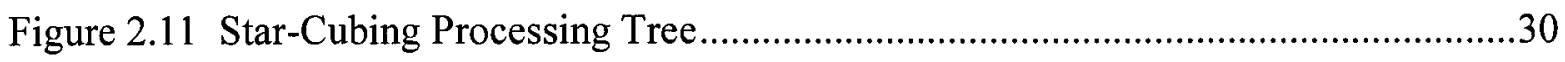

Figure 2.12 An Example of a Star Table and a Star Tree .................................................31

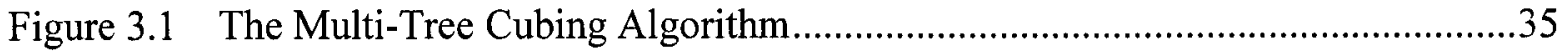

Figure 3.2 Multi-Tree Cubing Processing Tree..............................................................36

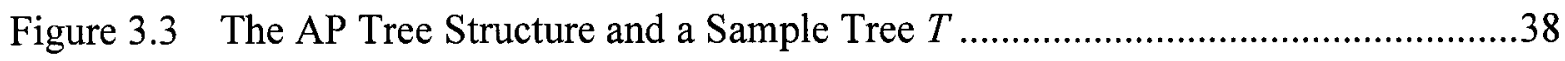

Figure 3.4 Single Partitions and Tree $T_{3}$ for Attribute Month .......................................40

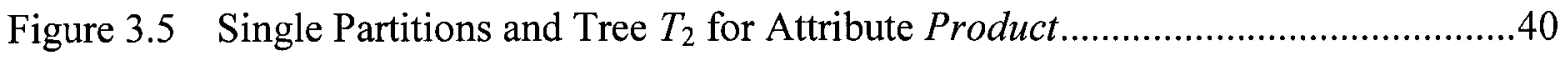

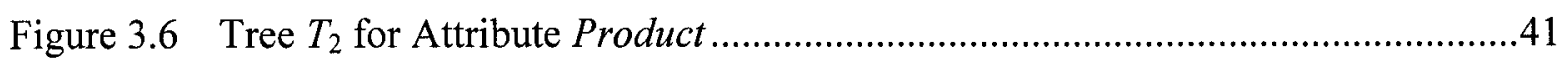

Figure 3.7 Tree $T_{1}$ for Attribute Salesperson..........................................................43

Figure 4.1 The User Interface of the Data Generator .................................................51 


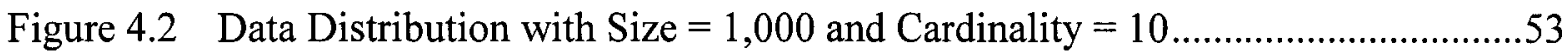

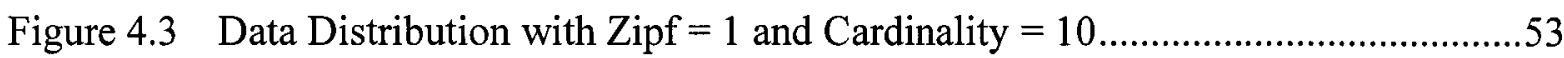

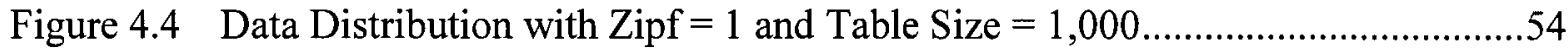

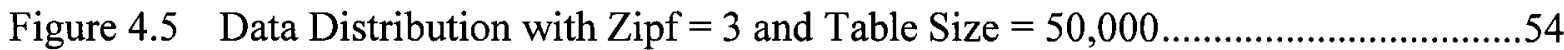

Figure 4.6 Table Size $($ Table $=$ Student, Dimension $=4$, MinSup $=1,000) \ldots \ldots \ldots \ldots \ldots \ldots \ldots . . .56$

Figure 4.7 Table Size $($ Table $=$ Student, Dimension $=4$, MinSup $=2,000) \ldots \ldots \ldots \ldots \ldots \ldots \ldots . . .56$

Figure 4.8 Table Size $($ Table $=$ PSCS, Dimension $=4$, MinSup $=500$, Zipf $=2) \ldots \ldots \ldots \ldots . .57$

Figure 4.9 Minimum Support $($ Table $=$ Student, Size $=20,000$, Dimension $=4) \ldots \ldots \ldots \ldots . .58$

Figure 4.10 Minimum Support $($ Table $=$ Student, $\operatorname{Size}=59,689$, Dimension $=4) \ldots \ldots \ldots \ldots . .58$

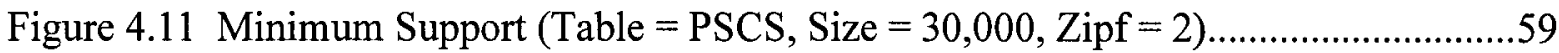

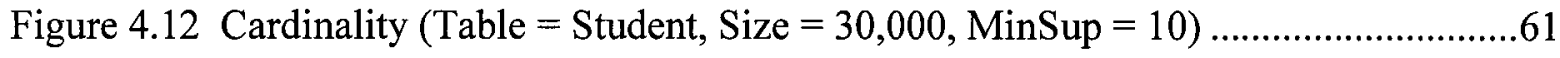

Figure 4.13 Cardinality $($ Table $=$ PSCS, Size $=20,000$, MinSup $=10$, Zipf $=0) \ldots \ldots \ldots \ldots \ldots \ldots . . .61$

Figure 4.14 Cardinality $($ Table $=$ PSCS, Size $=40,000, \operatorname{MinSup}=10, \mathrm{Zipf}=0) \ldots \ldots \ldots \ldots \ldots . . .62$

Figure 4.15 Dimension $($ Table $=$ Student, $\operatorname{Size}=30,000$, MinSup $=2,000) \ldots \ldots \ldots \ldots \ldots \ldots \ldots \ldots \ldots \ldots \ldots \ldots \ldots \ldots . . .63$

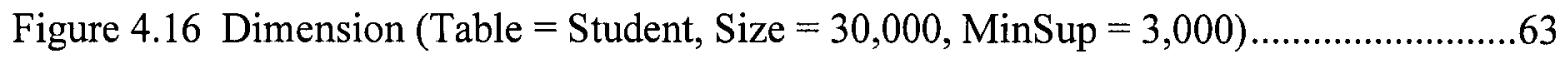

Figure 4.17 Data Skew $($ Table $=$ PSCS, Size $=20,000$, MinSup $=100) \ldots \ldots \ldots \ldots \ldots \ldots \ldots \ldots \ldots \ldots \ldots \ldots \ldots \ldots \ldots \ldots . . .64$

Figure 4.18 Data Skew $($ Table $=$ PSCS, Size $=40,000$, MinSup $=100) \ldots \ldots \ldots \ldots \ldots \ldots \ldots \ldots \ldots \ldots \ldots \ldots \ldots \ldots \ldots \ldots \ldots . . .65$ 


\section{CHAPTER 1}

\section{INTRODUCTION}

The problem addressed by this thesis lies within the research areas of on-line analytical processing, in general, and data cubes, in particular. These research areas are described in Sections 1.1 and 1.2, respectively. The organization of the remainder of this thesis is given in Section 1.3.

\subsection{On-line Analytical Processing}

Methods for performing sophisticated querying, analysis and reporting on large databases can suffer from long running times. Nonetheless, to improve their performance, organizations that have collected huge volumes of data often require that these kinds of analyses be performed to give them insights into their data. Thus, faster means of performing these types of analyses are of great interest to a wide community. In the past decade, on-line analytical processing (OLAP) tools have emerged as the most appropriate technique for accessing large databases [Berry and Linoff 1997]. OLAP tools provide multidimensional access, more detailed analysis, and faster performance; accordingly, corporations are now investing more in business intelligence software, including OLAP tools, than in on-line transaction processing applications (OLTP), such as Structured Query Language (SQL) querying. Historically, corporations spent more money on OLTP than OLAP [Oracle $9 i$ BI 2002]. 
The term "OLAP" was coined by Codd [Codd 1993], but it has been given various definitions by different research groups and individuals. A widely accepted short definition of $\boldsymbol{O L A P}$ is the "fast analysis of shared multidimensional information" [Creeth and Pendse 1995]. Let us consider several of the key words in this definition in more detail. The word "multidimensional" is present in this definition, because the attributes of the database are treated similarly to the dimensions of an array. In fact, OLAP is also referred to as multi-dimensional analysis. We can access the information relevant to a particular value for an attribute by using the value as an index along the relevant dimension. If the information to be accessed is related to $n$ values for each of $m$ attributes, we can use the $n$ values as indices along the $m$ relevant dimensions. When $m$ equals 1 or 2 , multi-dimensional analysis becomes equivalent to OLTP in the form of traditional SQL querying, which typically retrieves data at a detailed level. When $m$ is greater than 2, multi-dimensional analysis is performed using OLAP tools, which retrieve data at an aggregated level. The word "analysis" in the definition refers to the many analytical functions supported by OLAP tools, such as ranking functions, aggregation functions, and lagging/leading functions [Oracle 9i OLAP 2001]. For example, in the OLAP query "Who are the top 10 salespersons per region for the last two years?", a ranking function is needed to identify the "top" salespersons. The word "fast" refers to the quick response time provided by OLAP tools, which is typically only a few seconds, in contrast to the longer time required to design and run database queries on an online transaction processing system using a language such as SQL.

OLAP is closely related to data mining. Both data mining and OLAP have been widely and successfully applied in the field of knowledge discovery in databases (KDD). 
Data mining refers to discovering interesting patterns hidden in data with very limited domain knowledge from users [Fayyad et al. 1996]. Once patterns are generated, users need to examine them for usefulness. OLAP can find patterns and trends hidden within data as well. However, in addition to source data, OLAP also takes into account domain knowledge provided by domain experts. The users first supply a rough idea of what they are looking for, and then they use OLAP tools to discover the patterns. Therefore, OLAP can be thought of as a supervised form of data mining.

Two relatively new OLAP techniques, data cubes and iceberg cubes, play a central role in this thesis. They are discussed in the next section.

\subsection{Data Cubes}

The data cube (in the form of the CUBE BY operator) was introduced by Gray et al. [Gray et al. 1996] to improve the performance of OLAP. The CUBE BY operator generalizes the GROUP BY operator commonly found in relational database systems to compute all possible group-by aggregates of a list of attributes. The result of such a computation is called a data cube. Each dimension of a data cube represents one attribute in the list of attributes. In standard mathematical terminology, a data cube would be more correctly referred to as a hypercube, rather than a cube, because the number of dimensions is not limited to 3 . Each cell of the data cube contains some aggregate value, which can be Count, Total, Average, Minimum, Maximum, etc.

To compute a data cube from some other representation of data means to determine the aggregate value for each of the data cube's cells. The data cube can either be computed in advance of any query, which is called pre-computation, or relevant cells 
can be computed on the fly as needed to answer queries. If adequate memory is available to store the cube, pre-computation is more efficient if many OLAP queries are performed on the same data cube.

From an end-user's point of view, data cubes can be sliced and diced as needed. A slice operation performs a selection on one dimension and a dice operation performs a selection on two or more dimensions. Thus, slicing and dicing can be regarded as specifying conditions or attribute values that can be used to identify the cells of interest to the user.

Since every tuple of a table in a relational database is mapped to exactly one cell of the corresponding data cube, we say that the table is an extensional representation of the data cube. An SQL query with a CUBE BY operator specifies the calculation of all possible groups-by in the corresponding data cube, so we say it is an intensional representation of the data cube. In the context of data cubes, a group-by is a combination of attributes, such as $A, B C$, or $A C D$. Every distinct value of a group-by is called a partition. For example, if possible values for attributes $A, C$, and $D$ include $a_{1}, c_{7}$, and $d_{3}$, respectively, then $a_{1}-c_{7}-d_{3}$ is a partition for the $A C D$ group-by. We also use the word partition to describe the process of obtaining all of the partitions of a group-by. The computation of a data cube can be very expensive, because its cost grows exponentially with the number of attributes and linearly with the cardinalities of attributes [Hamilton \& Findlater 2003].

Consideration of the high cost of computing a data cube led to the definition of the iceberg cube problem [Beyer and Ramakrisnan 1999]. An iceberg cube contains exactly the partitions of all possible groups-by of a list of attributes that meet a certain 
aggregate condition. For example, an iceberg cube with a condition that a partition contains at least $N$ tuples can be expressed by the SQL query shown in Figure 1.1. The third line of this query is the CUBE BY clause, which indicates that three dimensions are required in the data cube.

\begin{tabular}{|ll|}
\hline SELECT & A, B, C, COUNT $\left({ }^{*}\right), \operatorname{SUM}(\mathrm{X})$ \\
FROM & $\mathrm{R}$ \\
CUBE BY & A, B, C \\
HAVING & $\operatorname{COUNT}\left({ }^{*}\right)>=N$ \\
\hline
\end{tabular}

Figure 1.1: Specification of a Sample Iceberg Cube

The assumption behind iceberg cubes is that users are only interested in group-by partitions that satisfy an aggregate condition. Therefore, an iceberg cube can be created without computing the group-by partitions that do not satisfy the aggregate condition. The aggregate condition is a small amount of domain knowledge that allows the creation of faster algorithms. Another assumption made is that the iceberg cube will require only a small subset of the data that is in the full data cube, like the "tip of the iceberg". In practice, these assumptions are valid, because the tuples present in a typical database represent only a tiny fraction of the possible tuples given the domains of the attributes. For example, if a database includes information about 10,000 people, 500 types of cars, and 20 financing plans, only a small fraction of the $10,000 \times 500 \times 20=100,000,000$ possible combinations of these values will actually need to be represented in the database. Where these assumptions are appropriate, finding only the iceberg cube instead of the full data cube greatly improves the performance of data cube computation. 
In this thesis, a new algorithm, called Multi-Tree Cubing, is proposed for computing iceberg cubes. By balancing the number of passes required over the database table against the amount of memory required to store intermediate data, this algorithm appears to have the potential to achieve superior performance to previous algorithms.

\subsection{Organization of the Thesis}

The remainder of this thesis is organized as follows.

Chapter 2 states the iceberg cube problem and describes three major approaches that well represent previous research. The three approaches are top-down, bottom-up and integrated top-down and bottom-up. Four algorithms belonging to the three approaches are reviewed.

Chapter 3 presents the Multi-Tree Cubing algorithm (MTC), which is an integrated top-down and bottom-up approach. The Multi-Tree Cubing algorithm uses a prefix tree data structure for sorting and processing. It is able to calculate multiple orderings simultaneously and prune partitions at the attribute level. An example of how the MTC algorithm works is given in this chapter.

Chapter 4 describes the five series of experiments that were designed to compare Multi-Tree Cubing with the Bottom-Up Computation (BUC), a well-known bottom-up approach. Both real and synthetic data were used in these experiments. The data generator that was used for preparing test data for these experiments is also described in this chapter. The experimental results showed that the Multi-Tree Cubing algorithm performed the same as or better than the BUC algorithm in all five series of experiments. 
Chapter 5 summarizes the thesis, draws conclusions, and then identifies the contributions of the thesis. Suggestions for future research are also discussed in this chapter. 


\section{CHAPTER 2}

\section{ICEBERG CUBE COMPUTATION}

In this chapter, we describe the computation of iceberg cubes. In Section 2.1, we describe data cubes and iceberg cubes and present examples of each. In Section 2.2, we describe the iceberg cube problem. Four algorithms that solve the problem are reviewed and compared in Section 2.3. As previously stated, the four algorithms fall into three categories: the top-down approach, the bottom-up approach, and the integrated top-down and bottom-up approach.

\subsection{Introduction}

An example database table called the Sales table is shown in Table 2.1 In the Sales table, the rows are referred to as tuples and the columns as attributes.

The Sales table shown in Table 2.1 can be represented by the three-dimensional data cube shown in Figure 2.1. Each dimension of the cube represents an attribute of the Sales table, and each cell represents a combination value of the three attributes, denoted by $(p, s, m)$, where $p$ is a particular product, $s$ is a particular salesperson, and $m$ is a particular month. In each cell, we store an aggregate value called the measure, which is computed from all tuples in the table containing product $p$, salesperson $s$, and month $m$. The measure can be a function such as Count, Sum, Average, Minimum, or Maximum. As a result, every tuple is mapped to exactly one cell and duplicate tuples in the database table are mapped to the same cell. In Figure 2.1, we use Count as the measure. 
Table 2.1: The Sales Database Table

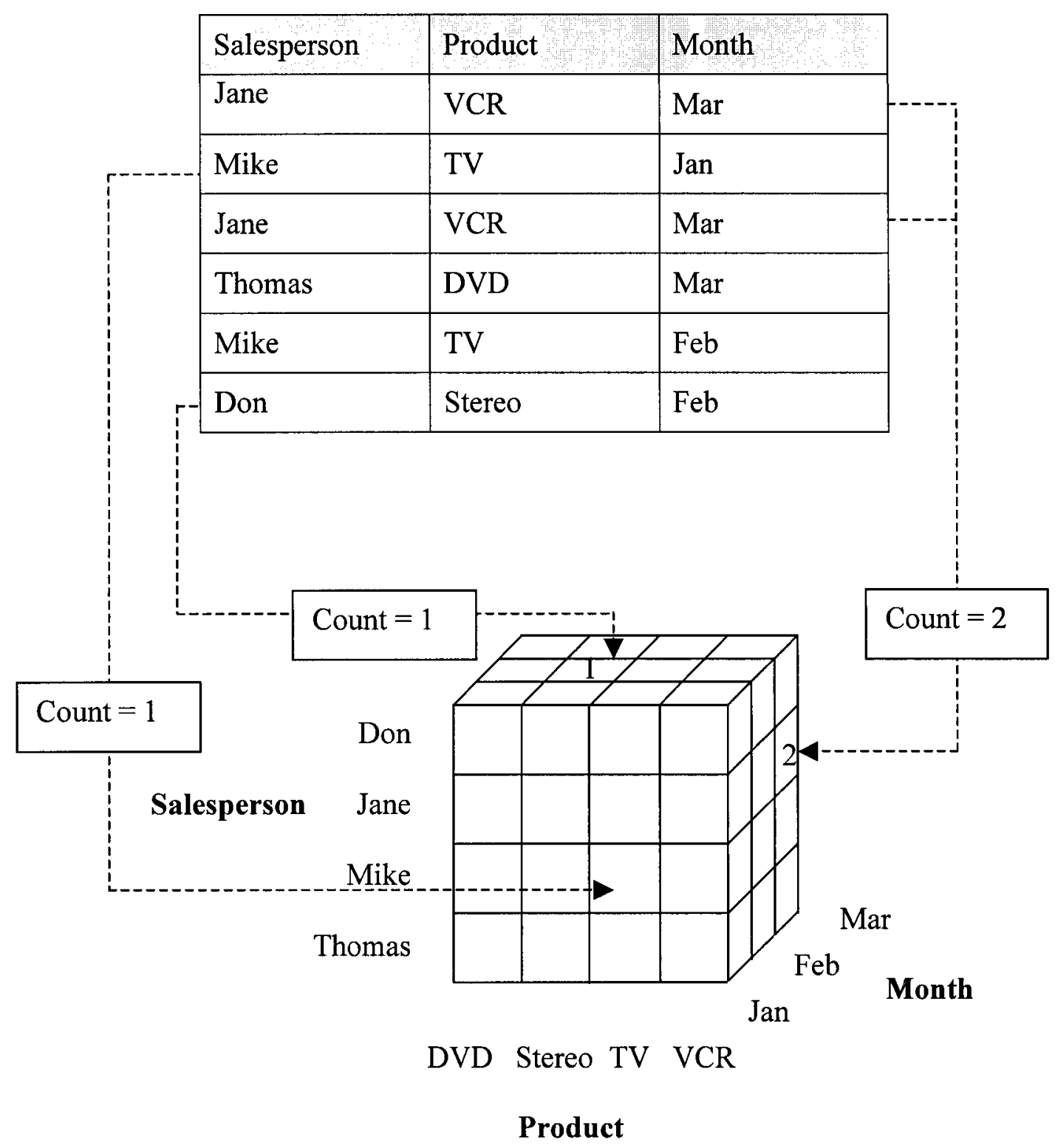

Figure 2.1: A 3-Dimensional Data Cube

The four unique values of attribute Salesperson, namely: Don, Jane, Mike, and Thomas, form the dimension Salesperson. Similarly, we form the other two dimensions: Product and Month. The first and the third tuples are duplicates, so they are mapped to the same cell, (Jane, VCR, Mar), which is the intersection of these three values across the 
three dimensions. Since Count is the measure, this cell stores a count of 2 . The second tuple is mapped to cell (Mike, TV, Jan), which has a count of 1 . Most cells in this data cube have no tuples mapped to them, so their counts are set to 0 . To simplify the diagram, we do not show the measure value if it is 0 . In addition, there are cells with nonzero counts that cannot be seen in Figure 2.1, such as the cell (Thomas, DVD, Mar), which has a count of 1 .

The corresponding query for the data cube in Figure 2.1 is shown in Figure 2.2. In the first line of this query, "COUNT $\left(^{*}\right)$ " indicates that Count is the measure. In the third line, the attributes corresponding to the dimensions of the data cube are listed.

\begin{tabular}{|ll|}
\hline SELECT & Salesperson, Product, Month, COUNT(*) \\
FROM & Sales \\
CUBE BY & Salesperson, Product, Month \\
\hline
\end{tabular}

Figure 2.2: Data Cube Query

In general for $m$ attributes, there are $2^{m}$ groups-by. The query shown in Figure 2.2 calculates $2^{3}$ or 8 groups-by based on 8 possible combinations of the three attributes in the database table. The 8 groups-by are Salesperson, Salesperson-Product, SalespersonProduct-Month, Salesperson-Month, Product, Product-Month, Month, and none (which represents the overall total of the measure values). The Count measure requires the calculation of the number of occurrences of each group-by partition. Using the Sales table shown in Table 2.1 as the input table, the output records for computing the data cube query shown in Figure 2.2 are given in Table 2.2. Among the 30 output records for the 7 groups-by, there are 19 with a count of 1 . 
Table 2.2: The Computed Data Cube

\begin{tabular}{|c|c|c|}
\hline Group-by & Partition & Count \\
\hline Salesperson & Don & 1 \\
\hline Salesperson & Jane & 2 \\
\hline Salesperson & Mike & 2 \\
\hline Salesperson & Thomas & 1 \\
\hline Salesperson-Product & Don-Stereo & 1 \\
\hline Salesperson-Product & Jane-VCR & 2 \\
\hline Salesperson-Product & Mike-TV & 2 \\
\hline Salesperson-Product & Thomas-DVD & 1 \\
\hline Salesperson-Product-Month & Don-Stereo-Feb & 1 \\
\hline Salesperson-Product-Month & Jane-VCR-Mar & 2 \\
\hline Salesperson-Product-Month & Mike-TV-Feb & 1 \\
\hline Salesperson-Product-Month & Mike-TV-Jan & 1 \\
\hline Salesperson-Product-Month & \begin{tabular}{|l|} 
Thomas-DVD-Mar \\
\end{tabular} & 1 \\
\hline Salesperson-Month & Don-Feb & 1 \\
\hline Salesperson-Month & Jane-Mar & 2 \\
\hline Salesperson-Month & Mike-Feb & 1 \\
\hline Salesperson-Month & Mike-Jan & 1 \\
\hline Salesperson-Month & Thomas-Mar & 1 \\
\hline Product & DVD & 1 \\
\hline Product & Stereo & 1 \\
\hline Product & TV & 2 \\
\hline Product & VCR & 2 \\
\hline Product-Month & DVD-Mar & 1 \\
\hline Product-Month & Stereo-Feb & 1 \\
\hline Product-Month & TV-Feb & 1 \\
\hline Product-Month & \begin{tabular}{|l|} 
TV-Jan \\
\end{tabular} & 1 \\
\hline Product-Month & VCR-Mar & 2 \\
\hline Month & Feb & 2 \\
\hline Month & Jan & 1 \\
\hline Month & Mar & 3 \\
\hline
\end{tabular}

CUBE BY and GROUP BY queries are closely related to each other. We have already seen an example of a CUBE BY query. Next, an example of a GROUP BY query 
is shown in Figure 2.3. Using the Sales table shown in Table 2.1 as the input table, the output records for computing this example GROUP BY query are shown in Table 2.3. Only the partitions for one group-by Salesperson-Product-Month are computed and output for the GROUP BY query, while the partitions for 8 groups-by are computed and output for the CUBE BY query.

\begin{tabular}{|ll|}
\hline SELECT & Salesperson, Product, Month, COUNT( $\left.{ }^{*}\right)$ \\
FROM & Sales \\
GROUP BY & Salesperson, Product, Month \\
\hline
\end{tabular}

Figure 2.3: An Example of a GROUP BY Query

Table 2.3: Results for the Example GROUP BY Query

\begin{tabular}{|l|l|c|}
\hline Group-by & Partition & Count \\
\hline Salesperson-Product-Month & Don-Stereo-Feb & 1 \\
\hline Salesperson-Product-Month & Jane-VCR-Mar & 2 \\
\hline Salesperson-Product-Month & Mike-TV-Jan & 1 \\
\hline Salesperson-Product-Month & Mike-TV-Feb & 1 \\
\hline Salesperson-Product-Month & Thomas-DVD-Mar & 1 \\
\hline
\end{tabular}

\subsection{Problem Statement}

The data cube problem is to compute a specified measure for all cells in the data cube. The goal of much research on data cubes is to solve this problem as efficiently as possible. If the data cube has many dimensions, existing methods for this problem are both time consuming and memory intensive.

The iceberg cube problem is to compute a specified measure for all group-by partitions with a measure value greater than or equal to a threshold called the minimum support threshold (or MinSup) [Beyer and Ramakrishnan 1999]. For the Sales table 
shown in Table 2.1, with Count as the measure and 10 as the minimum support threshold, we have the iceberg cube query shown in Figure 2.4. The corresponding data cube can be visualized as similar to that shown in Figure 2.1, but with all cells with measure values less than 10 removed. If the threshold has been appropriately chosen, this iceberg cube can be used to answer questions such as "Which products are the best sellers in each month?" or "Who are the top three salespeople in each month?", because all required information is available in the iceberg cube. However, this iceberg cube cannot be used to identify all products that Thomas has sold at least one of, because the threshold used was 10 .

\begin{tabular}{|ll|}
\hline SELECT & Salesperson, Product, Month, COUNT $\left(^{*}\right)$ \\
FROM & Sales \\
CUBE BY & Salesperson, Product, Month \\
HAVING & COUNT $\left({ }^{*}\right)>=10$ \\
\hline
\end{tabular}

Figure 2.4: Iceberg Cube Query (MinSup = 10)

Since the Sales table shown in Table 2.1 has only 6 tuples, the iceberg cube generated in response to the iceberg cube query shown in Figure 2.4 would be empty, because in this case, no partition satisfies the threshold of 10 . To illustrate a non-empty iceberg cube, we use 2 as the minimum support threshold, which gives the iceberg cube query shown in Figure 2.5. We will use this iceberg cube query as an example extensively throughout this thesis. All algorithms that answer this iceberg cube query should yield the output records shown in Table 2.4, although the order of the records may vary. Answering an iceberg cube query is also referred to as computing the iceberg cube. 


\begin{tabular}{|ll|}
\hline SELECT & Salesperson, Product, Month, COUNT $\left(^{*}\right)$ \\
FROM & Sales \\
CUBE BY & Salesperson, Product, Month \\
HAVING & COUNT $\left({ }^{*}\right)>=2$ \\
\hline
\end{tabular}

Figure 2.5: Iceberg Cube Query (MinSup = 2)

Table 2.4: The Computed Iceberg Cube (MinSup = 2)

\begin{tabular}{|l|l|c|}
\hline Group-by & Partition & Count \\
\hline Salesperson & Jane & 2 \\
\hline Salesperson-Product & Jane-VCR & 2 \\
\hline Salesperson-Product-Month & Jane-VCR-Mar & 2 \\
\hline Salesperson & Mike & 2 \\
\hline Salesperson-Product & Mike-TV & 2 \\
\hline Product & VCR & 2 \\
\hline Product & TV & 2 \\
\hline Product-Month & VCR-Mar & 2 \\
\hline Month & Mar & 3 \\
\hline Month & Feb & 2 \\
\hline
\end{tabular}

A pre-computed iceberg cube is also useful for answering iceberg queries [Fang et al. 1998]. An iceberg query computes a single group-by for any tuples that satisfy the minimum support condition. For example, the iceberg query shown in Figure 2.6 computes the group-by Salesperson-Product-Month for any partition with a Count of 2 or more. We can easily see from Table 2.4 that the result for this iceberg query is JaneVCR-Mar with a Count of 2. 


\begin{tabular}{|ll|}
\hline SELECT & Salesperson, Product, Month, COUNT $\left(^{*}\right)$ \\
FROM & Sales \\
GROUP BY & Salesperson, Product, Month \\
HAVING & COUNT $\left({ }^{*}\right)>=2$ \\
\hline
\end{tabular}

Figure 2.6: A Sample Iceberg Query

\subsection{Iceberg Cube Algorithms}

Algorithms for computing iceberg cubes are described in this subsection. The basis for the algorithms is described in section 2.3.1. Then, four algorithms namely TopDown Computation, Multi-Way Array, Bottom-Up Computation, and Star Cubing, are described in the remaining four subsections.

\subsubsection{The Basis of the Algorithms}

Figure 2.7 shows a 4-dimensional lattice that includes all of the groups-by for four attributes: A, B, C and D. Each node represents one group-by and each arc represents a parent/child relationship between two groups-by. The node at the lower level represents the parent group-by and the node at the higher level represents the child group-by. For example, group-by $A$ is the parent of group-by $A B$.

To compute an iceberg cube, all partitions of groups-by with a measure value above a specified threshold must be identified. A simple algorithm for this problem is to compute each group-by separately. For the case shown in Figure 2.6, which has 4 attributes, this algorithm requires $2^{4}$ or 16 scans over the database table to calculate the 16 groups-by. For large databases, with perhaps hundreds of millions of tuples, the 
input/output time required to perform each scan may be considerable. Thus, the simple algorithm is not efficient, because it computes the measure values for only a single group-by during each pass.

Several algorithms have been proposed for computing the iceberg cube more efficiently than the simple algorithm just discussed. These algorithms output the same iceberg cube, although the order of the output records may vary, as previously mentioned.

The goal of an efficient algorithm is to take advantage of as much commonality as possible between a parent and child [Beyer and Ramakrisnan 1999]. One commonality that can be exploited for efficiency occurs when a parent and a child share a prefix, which is the initial subsequence of the attribute names. For example, parent group-by $A$ and child group-by $A B$ share the same prefix $A$. The techniques for exploiting this shared prefix used by previous algorithms can be divided into two categories, namely Apriorilike pruning and shared computation.

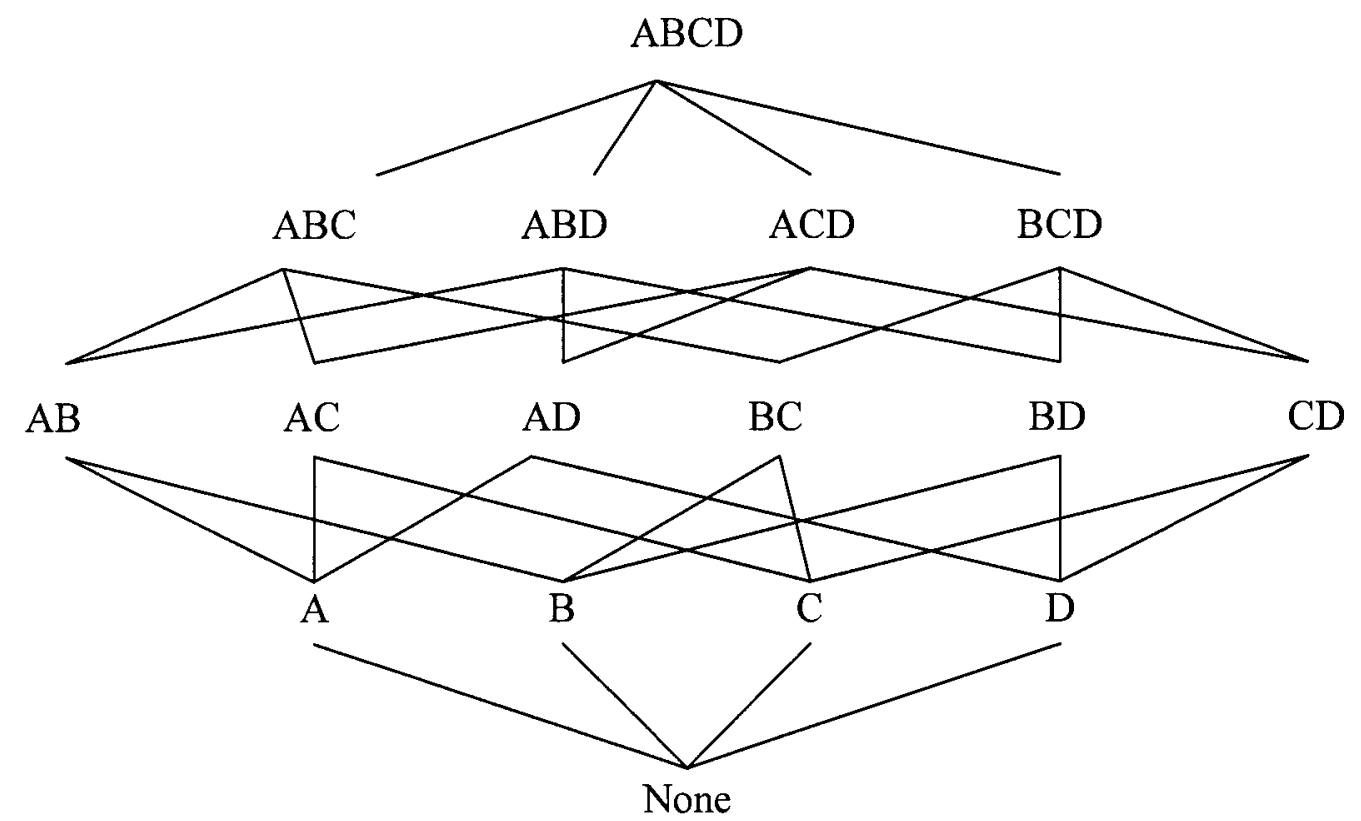

Figure 2.7: A 4-Dimensional Lattice 
Apriori-like pruning is based on the anti-monotonic property of a pruning function. The term "Apriori-like" refers to the Apriori algorithm [Agrawal and Srikant 1994], where this type of pruning was exploited to compute combinations of items efficiently. Given function $f$, some value $v$, and two partitions $A$ and $A B$ such that $A B \subseteq A$, if $f(A)<=v$, then $f(A B)<=v$ is always true, then the function $f$ is anti-monotonic [Han et al. 2001]. Measures such as Count, Sum, Minimum, and Maximum are anti-monotonic, so they can be directly used in Apriori-like pruning. For example, for $a_{i} \in A$, if $f\left(a_{i}\right)$ does not satisfy the minimum support threshold, then we can safely prune all $a_{i} b_{i}$ such that $b_{i} \in$ $B$, because they will also definitely not satisfy the minimum support threshold. Apriorilike pruning is used in the Bottom-Up Computation algorithm described in Section 2.3.3.

Shared computation refers to the calculation of the measure values for two or more groups-by during the same pass over the database. Because of the shared prefix between parent and child groups-by, when we partition the child group-by, we can calculate the measure value for parent and child at the same time. This is the main idea behind top-down approaches, such as the Top-Down Computation algorithm described in Section 2.3.2.

Another form of shared computation involves making use of partial totals. For the data cube problem, as opposed to the iceberg cube problem, partial totals (marginal totals) can be used to speed up the computing of grand totals. For example, given Count as the measure, for $a_{i} \in A$ for some parent group-by $A$, we can calculate $\operatorname{Count}\left(a_{i}\right)$ by adding up all of the $\operatorname{Count}\left(a_{i} b_{i}\right)$ values such that $b_{i} \in B$ for some child group-by $B$. Alternatively, we can also calculate $\operatorname{Count}(\mathrm{A})$ from $\operatorname{Count}(\mathrm{AC})$ or $\operatorname{Count}(\mathrm{AD})$. This technique for using partial totals is one of the cornerstones of the Multi-Way Array 
algorithm described in Section 2.3.4. We were unable to create an effective strategy for using partial totals for the iceberg cube problem.

\subsubsection{Top-Down Computation}

As the name implies, the Top-Down Computation (TDC) algorithm [Hamilton and Findlater 2003] is a top-down approach that starts from the least aggregated groupsby at the top of lattice and works its way down to the most aggregated groups-by at the bottom. For a data cube with four dimensions $A, B, C$, and $D$, the corresponding TDC processing tree is shown in Figure 2.8. Each underlined group-by is also an ordering, i.e., a child group-by that permits the shared computation of its parent and other ancestor groups-by during the pass in which it is being computed. By using orderings, the number of passes over the database can be reduced. In Figure 2.8, the numbers preceding the underlined groups-by give the order of processing.

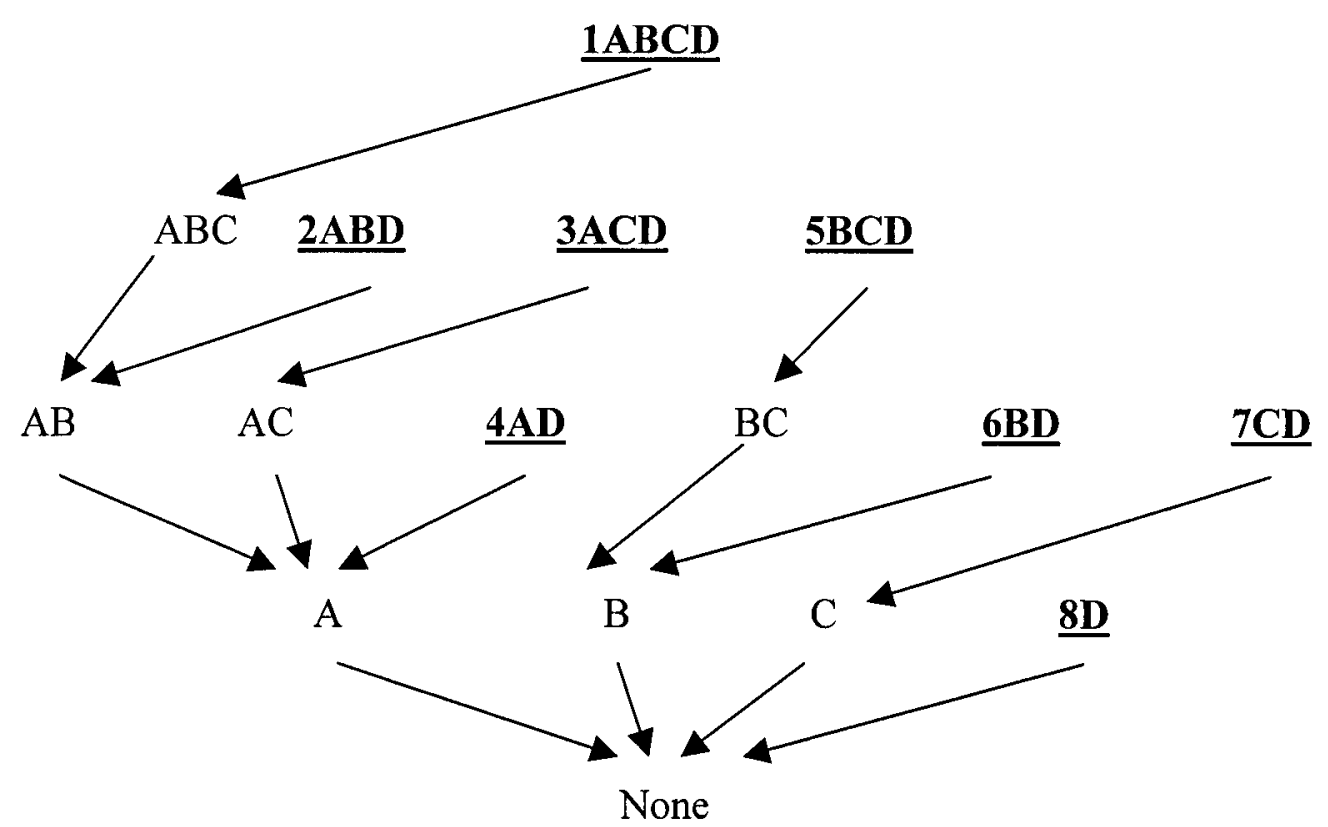

Figure 2.8: The TDC Processing Tree 
TDC uses orderings to cover all of the groups-by. To cover the $2^{m}$ groups-by of an $m$-dimensional data cube, $2^{m-1}$ orderings are required [Hamilton and Findlater 2003]. When processing an ordering, TDC partitions the data using it and simultaneously calculates all groups-by that are prefixes of it. For example, to partition ordering $A B C D$, TDC calculate four groups-by at the same time: $A B C D, A B C, A B$, and $A$, because each of them is a prefix of $A B C D$. Similarly, when we partition the next ordering $A B D$, we can calculate $A B D, A B$, and $A$ simultaneously. However, since we have already computed $A B$ and $A$, we do not calculate them again. Shared computation is the main advantage of TDC.

TDC's computation for the iceberg cube query shown in Figure 2.5 is summarized in Table 2.5. During the first pass, the ordering Salesperson-Product-Month is processed; using shared computation, its parent Salesperson-Product and its other ancestor Salesperson are also processed. Separate counts are maintained for each partition of these groups-by. For example, the numbers of occurrences of the DonStereo-Feb and Jane-VCR-March partitions of the Salesperson-Product-Month group-by are counted. At the end of the first pass, partitions with counts less than the threshold of 2 are pruned. Since Don-Stereo-Feb has a count of 1, it is pruned, while Jane-VCRMarch, with a count of 2, is output. During the second pass, the Salesperson-Month ordering is computed, and so forth.

The main disadvantage of TDC is weak pruning, i.e., it is relatively poor at identifying cases where pruning is possible. Two cases where pruning opportunities are missed are during the processing of the first ordering and between orderings. 
Table 2.5: Summary of the TDC Computation Process

\begin{tabular}{|c|l|l|c|l|}
\hline Scan & Group-by & Partition & Count & Output/Pruned \\
\hline 1 & Salesperson & Don & 1 & Pruned \\
\hline 1 & Salesperson-Product & Don-Stereo & 1 & Pruned \\
\hline 1 & Salesperson-Product-Month & Don-Stereo-Feb & 1 & Pruned \\
\hline 1 & Salesperson & Jane & 2 & Output \\
\hline 1 & Salesperson-Product & Jane-VCR & 2 & Output \\
\hline 1 & Salesperson-Product-Month & Jane-VCR-Mar & 2 & Output \\
\hline 1 & Salesperson & Mike & 2 & Output \\
\hline 1 & Salesperson-Product & Mike-TV & 2 & Output \\
\hline 1 & Salesperson-Product-Month & Mike-TV-Feb & 1 & Pruned \\
\hline 1 & Salesperson-Product-Month & Mike-TV-Jan & 1 & Pruned \\
\hline 1 & Salesperson & Thomas & 1 & Pruned \\
\hline 1 & Salesperson-Product & Thomas-DVD & 1 & Pruned \\
\hline 1 & Salesperson-Product-Month & Thomas-DVD-Mar & 1 & Pruned \\
\hline 2 & Salesperson-Month & Don-Feb & 1 & Pruned \\
\hline 2 & Salesperson-Month & Jane-Mar & 2 & Output \\
\hline 2 & Salesperson-Month & Mike-Feb & 1 & Pruned \\
\hline 2 & Salesperson-Month & Mike-Jan & 1 & Pruned \\
\hline 2 & Salesperson-Month & Thomas-Mar & 1 & Pruned \\
\hline 3 & Product & DVD & 1 & Pruned \\
\hline 3 & Product-Month & DVD-Mar & 1 & Pruned \\
\hline 3 & Product & Stereo & 1 & Pruned \\
\hline 3 & Product-Month & Stereo-Feb & 1 & Pruned \\
\hline 3 & Product & TV & 2 & Output \\
\hline 3 & Product-Month & TV-Feb & 1 & Pruned \\
\hline 3 & Product-Month & TV-Jan & 1 & Pruned \\
\hline 3 & Product & VCR & 2 & Output \\
\hline 3 & Product-Month & VCR-Mar & 2 & Output \\
\hline 4 & Month & Feb & 2 & Output \\
\hline 4 & Month & Man & 1 & Pruned \\
\hline 4 & Month & 3 & Output \\
\hline & & & \\
\hline
\end{tabular}


During the scan of the database to process its first ordering, TDC may perform a significant amount of useless work. For example, for the first ordering $A B C D$, TDC partitions the whole database using all of the attributes. For the four attributes $A, B, C$, and $D$, the most difficult case is $A B C D$, because it has the most partitions. TDC always tackles the hardest case during the processing of the first ordering, when it is accumulating totals for $A B C D, A B C, A B$, and $A$. A better pruning strategy would be to identify partitions $a_{i} \in A$ that do not satisfy the minimum support condition and then prune partitions $a_{i} b_{j}$ such that $a_{i} \in A$ and $b_{j} \in B$ for groups-by $A B, A B C$, and $A B C D$. Unfortunately, TDC lacks of this kind of pruning, so it always partitions the whole ordering, even though some of those partitions could be pruned.

Since TDC's pruning strategy is limited to pruning complete orderings, it cannot prune some of the partitions of related orderings. For example, suppose that no results are found for group-by $A$ after ordering $A B C D$ has been processed. Then, TDC is able to prune the orderings $A B D, A C D$, and $A D$, based on Apriori-like pruning. But, if only some partition of $A$ does not meet the minimum support condition, TDC's pruning strategy is not able to prune the corresponding partitions for other orderings. For example, if $a_{2} \in A$ fails the aggregate condition, a better pruning strategy would prune all partitions $a_{2} b_{i} d_{j}$ such that $b_{i} \in B$ and $d_{j} \in D$ for group-by $A B D$, which belongs to another ordering $A B D$. Again, TDC lacks this type of pruning.

\subsubsection{The Multi-Way Array Algorithm}

The Multi-Way Array algorithm [Zhao et al. 1997] is a top-down approach that loads input data with $n$ attributes into $n$-dimensional array chunks for processing. An 
apparently straightforward approach to representing a data cube for $n$ attributes is to simply have an array with $n$ dimensions. However, the occurring values for an attribute may be widely scattered and the total size of the array may be too large to fit in memory. The Multi-Way Array algorithm addresses these problems by mapping values into condensed ranges and processing the arrays in chunks.

To map values into a condensed range, the Multi-Way Array algorithm needs to know the cardinalities of attributes in advance. The cardinality of an attribute refers to the number of distinct values for it that appear in the database. The Multi-Way Array algorithm takes the $n$ values from a tuple and converts them into $n$ integers between 0 and one less than the cardinality. For example, if the cardinality of one of the attributes was 3 and the values that actually occurred in the database are $17,6,266$, and 1,000,001, they would be mapped into 0,1 , and 2 . Likewise, month names would be sorted in alphabetic order and converted to values 0 to 11 . Then, it uses the $n$ integers as indices of the $n$ dimensional array to locate the cell and adds one to the count of the cell. So, the $n$ dimensional array stores a count in each cell and the indices of the cell are mapped to a particular tuple. The count in a cell indicates the number of corresponding tuples in the table. Since the data is completely stored in the array, the computation of the Multi-Way algorithm requires no tuple comparison and is driven only by the array indices. For example, using the Sales tables shown in Table 2.1, the Multi-Way Array algorithm creates a 3-dimensional array to store the data for computing the iceberg cube query shown in Figure 2.5. Since it is a 3-dimensional array, we use Salesperson as the first index and Product and Month as the other two indices, and show a 2-dimensional array for each salesperson in Tables 2.6. In Sales table shown in Table 2.1, there is only one 
tuple for salesperson Don, so the cell (Don, Stereo, Feb) in Table 2.6(a) has a count of 1 and the other cells all have counts of 0 . A similar pattern is observed for the other salespersons, as shown in Table 2.6(b) - (d).

Table 2.6: 2-Dimenisonal Arrays for Four Partitions (a) A 2-Dimenisonal Array for Salesperson: Don

\begin{tabular}{|c|c|c|c|c|}
\hline Month/Product & DVD & Stereo & TV & VCR \\
\hline Feb & 0 & 1 & 0 & 0 \\
\hline Jan & 0 & 0 & 0 & 0 \\
\hline Mar & 0 & 0 & 0 & 0 \\
\hline
\end{tabular}

(b) A 2-Dimenisonal Array for Salesperson: Jane

\begin{tabular}{|c|c|c|c|c|}
\hline Month/Product & DVD & Stereo & TV & VCR \\
\hline Feb & 0 & 0 & 0 & 0 \\
\hline Jan & 0 & 0 & 0 & 0 \\
\hline Mar & 0 & 0 & 0 & 2 \\
\hline
\end{tabular}

(c) A 2-Dimenisonal Array for Salesperson: Mike

\begin{tabular}{|c|c|c|c|c|}
\hline Month/Product & DVD & Stereo & TV & VCR \\
\hline Feb & 0 & 0 & 1 & 0 \\
\hline Jan & 0 & 0 & 1 & 0 \\
\hline Mar & 0 & 0 & 0 & 0 \\
\hline
\end{tabular}

(d) A 2-Dimenisonal Array for Salesperson: Thomas

\begin{tabular}{|c|c|c|c|c|}
\hline Month/Product & DVD & Stereo & TV & VCR \\
\hline Feb & 0 & 0 & 0 & 0 \\
\hline Jan & 0 & 0 & 0 & 0 \\
\hline Mar & 1 & 0 & 0 & 0 \\
\hline
\end{tabular}

Two issues with the $n$-dimensional array used by the Multi-Way algorithm are that it may grow too large to fit into main memory and that many cells in the array are 
unneeded (with a count of 0 ). To address the first issue, the $n$-dimensional array is divided into small size $n$-dimensional array chunks and each chunk is small enough to comfortably fit into memory. Each array chunk is loaded into memory only once. For the other issue, Multi-Way applies a sparse array technique, which only represents nonempty cells; each such cell is stored as a pair of the cell position and its count.

Shared computation is the most important feature of this algorithm. Figure 2.9 shows its processing tree for four attributes. In the processing tree, we can roll up the intermediate results for group-by $A B C$ along attribute $C$ and get the results for group-by $A B$. For example, if group-by $A B C$ includes three partitions $a_{1} b_{2} c_{1}, a_{1} b_{2} c_{5}$, and $a_{1} b_{2} c_{9}$, with counts of 10,15 , and 40 , respectively, the algorithm rolls up these partitions to form $a_{1} b_{2}$ with a count of 65 . Similarly, we can get the results for $A C$ and $B C$ from $A B C$.

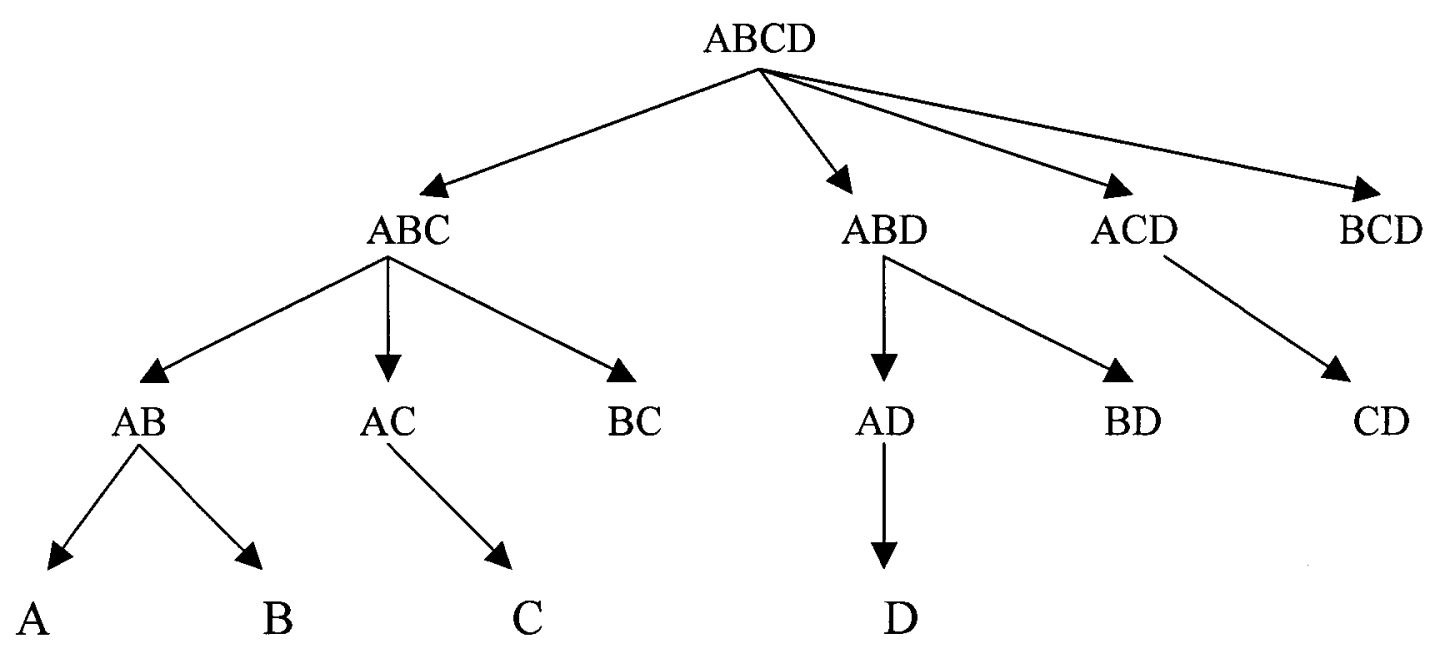

Figure 2.9: Multi-Way Array Processing Tree

Zhao et al. proved that sorting the attributes in cardinality ascending order requires the minimum amount of memory during computation. Suppose the cardinalities for attributes $A, B, C$, and $D$ are $10,100,1000$, and 10000 . To process these attributes in cardinality ascending order, we first process $A$, then $B$, etc. According to their 
calculations, the total memory required for cardinality ascending order is $4 \mathrm{MB}$, while the total memory required for cardinality descending order is 4 GB [Zhao et al. 1997].

The Multi-Way Array algorithm was originally designed for the data cube problem, so if it is applied to the iceberg cube problem, it does not perform any pruning while computing the iceberg cube. For a sparse database, this algorithm can compress the data to a certain degree, but, if the database is too sparse, the arrays will grow too large to fit into main memory. Due to its unique array-based design, the Multi-Way Array algorithm is one of the best performers for computing the data cube, especially when the cardinality is moderate and the database is dense. It is less effective for the iceberg cube problem, because of its lack of pruning and its high memory usage.

\subsubsection{Bottom-Up Computation}

The Bottom-Up Computation (BUC) algorithm is a bottom-up approach [Beyer and Ramakrishnan 1999]. BUC processes the partitions starting from a single attribute and moves towards the apex of the lattice. Figure 2.10 shows a BUC processing tree for four attributes. The numbers preceding the groups-by indicate the processing order.

For the processing tree shown in Figure 2.10, BUC first partitions on attribute $A$ and checks the minimum support condition for the first partition $a_{1}$. If the condition is not satisfied, BUC prunes any partitions starting with $a_{1}$, which include $a_{1} b_{i}\left(b_{i} \in B\right), a_{1} c_{i}\left(c_{i}\right.$ $\in C)$, and $a_{1} d_{i}\left(d_{i} \in D\right)$, by stopping recursion. If the condition for $a_{1}$ is satisfied, BUC outputs $a_{1}$, then recursively moves on to the next group-by, which is $A B$. It partitions the attribute $B$ to compute new partitions $a_{1} b_{i}$, such that $b_{i} \in B$. If $a_{1} b_{i}$ satisfies the minimum support condition, then the next group-by, $\mathrm{ABC}$, is examined, and so forth. Once the processing for $a_{1}$ is complete, BUC continues checking the remaining partitions of $A$. At 
this point, all of the groups-by starting from attribute $A$ have been computed. Next, BUC starts from the remaining attributes $B, C$, and $D$ and repeats the process for each.

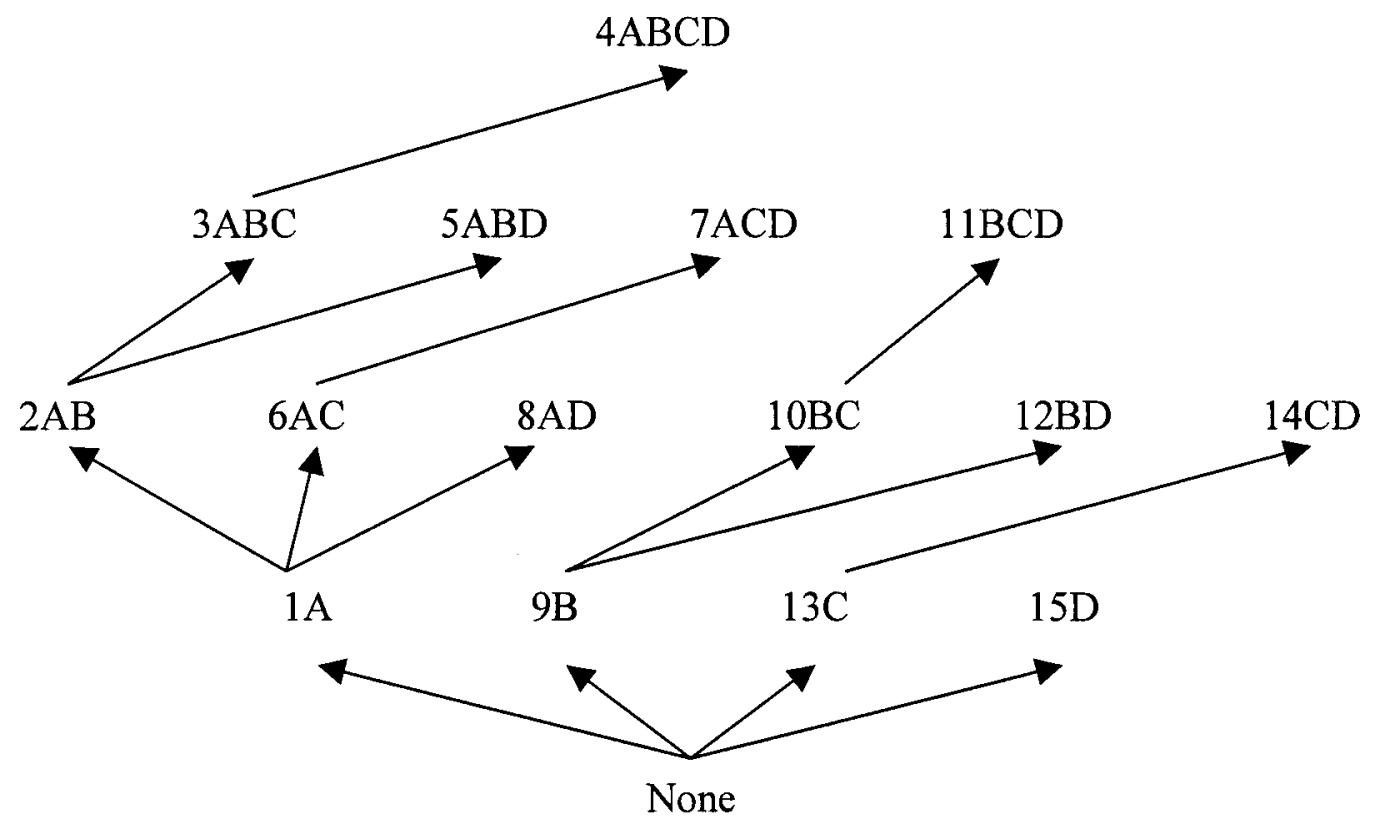

Figure 2.10: The BUC Processing Tree

The BUC computation process for computing the iceberg cube query shown in Figure 2.5 is summarized in Table 2.7. For the first pass, the database is sorted using Salesperson as the primary sort key. After sorting, Don is the first partition of Salesperson, assuming alphabetical ordering is used for the sorting. Next BUC checks whether the Don partition meets the minimum support condition, and if so, sorts the tuples in this partition using Product as a (secondary) sort key. In this case, since there is only one record in this partition, its Count is less than the threshold and thus it is pruned. Partitions of Salesperson-Product and Salesperson-Product-Month that have Don as the salesperson are not counted, since their counts will not reach the threshold. The second partition of Salesperson is Jane. After this partition is counted, its count is 2, which reaches the threshold. The records in this partition are sorted using Product as the sort 
key. Next, the partitions of Salesperson-Product that have Jane as the salesperson are examined. If necessary, they would be sorted using Month as a (tertiary) sort key. It happens that there is only one, namely, Jane-VCR, so its count is examined. Since its count is 2, it is output. The partitions of Salesperson-Product-Month that have Jane as salesperson and $V C R$ as product are examined. Again, there is only one, namely, JaneVCR-March with a count of 2, so it is output. Processing then continues with the third partition of Salesperson, which is Mike, and after that is completed, with the next individual attribute Product, and so forth.

From the example given in Table 2.7, we can observe that BUC relies on Apriorilike pruning to reduce the computation space. BUC is a divide and conquer strategy, and partitioning is its major cost. Partitioning is performed using a linear sorting method called CountingSort, which excels at sorting large lists with many duplicates. CountingSort requires that the sort key be an integer value between zero and its cardinality, and that the cardinality be known in advance. CountingSort stores the counts of a list of integers in an array and uses these integers as array indices. Every time CountingSort reads an integer, it uses the integer to locate its count in the array and increment the count by 1 .

For best performance, BUC requires that attributes be sorted in cardinality descending order. This ensures that the most discriminating attribute, which has the highest cardinality, is processed first. For example, if Product had 10,000 possible values, while Salesperson and Month had only 12 each, BUC would do best by first determining which of the products met the minimum support condition. Typically, there 
are only a few of them. Further processing could be limited to those few products in combination with the relatively few salespersons and months.

Table 2.7: Summary of BUC Computation Process

\begin{tabular}{|c|l|l|c|l|}
\hline Scan & Group-by & Partition & Count & Output/Pruned \\
\hline 1 & Salesperson & Don & 1 & Pruned \\
\hline 1 & Salesperson & Jane & 2 & Output \\
\hline 1 & Salesperson-Product & Jane-VCR & 2 & Output \\
\hline 1 & Salesperson-Product-Month & Jane-VCR-Mar & 2 & Output \\
\hline 1 & Salesperson & Mike & 2 & Output \\
\hline 1 & Salesperson-Product & Mike-TV & 2 & Output \\
\hline 1 & Salesperson-Product-Month & Mike-TV-Feb & 1 & Pruned \\
\hline 1 & Salesperson & Thomas & 1 & Pruned \\
\hline 2 & Salesperson-Month & Jane-Mar & 2 & Output \\
\hline 2 & Salesperson-Month & Mike-Feb & 1 & Pruned \\
\hline 2 & Salesperson-Month & Mike-Jan & 1 & Pruned \\
\hline 3 & Product & DVD & 1 & Pruned \\
\hline 3 & Product & Stereo & 1 & Pruned \\
\hline 3 & Product & TV & 2 & Output \\
\hline 3 & Product-Month & TV-Feb & 1 & Pruned \\
\hline 3 & Product-Month & TV-Jan & 1 & Pruned \\
\hline 3 & Product & VCR & 2 & Output \\
\hline 3 & Product-Month & VCR-Mar & 2 & Output \\
\hline 4 & Month & Feb & 2 & Output \\
\hline 4 & Month & Jan & 1 & Pruned \\
\hline 4 & Month & Mar & 3 & Output \\
\hline & & & & \\
\hline
\end{tabular}

BUC can be used to compute either a full data cube or an iceberg cube. Due to its pruning power, BUC works especially well at computing iceberg cubes for sparse database tables. As well, BUC is not memory intensive. When the database is dense, the 
partitioning costs more and the pruning is less effective, so the overall performance of BUC degrades. According to extensive studies, BUC performs better than TDC in most cases [Hamilton and Findlater 2003].

\subsubsection{The Star-Cubing Algorithm}

To integrate top-down and bottom-up approaches and obtain both of their benefits, the Star-Cubing algorithm was proposed [Xin et al. 2003]. Globally, Star-Cubing is a topdown approach and has TDC's major benefit, which is shared computation. To overcome TDC's weak pruning, Star-Cubing first determines the shared dimension between two groups-by, which is the prefix shared by the two groups-by. Then, it computes the two groups-by and applies Apriori-like pruning based on the results for the shared dimension. Locally, in the computation of the shared dimension, Star-Cubing acts as a bottom-up approach. Figure 2.11 shows a Star-Cubing processing tree with nodes labeled by their groups-by and their shared dimension with their child above. For example, the label $A B D / A B$ means that $A B D$ is the group-by and $A B$ is the shared dimension between $A B D$ and its parent group-by $A B C D$. To compute $A B C D$ and $A B D$, Star-Cubing first determines the shared dimension between them, which is $A B$. Then, it computes the two groups-by and prunes the partitions of group-by $A B C D$ using the partitions of group-by $A B D$ that do not satisfy the aggregate condition.

Two data structures, called star tables and star trees, were proposed for the StarCubing algorithm. A star table records one-dimensional partitions that do not satisfy the aggregate condition and therefore are replaced with an asterisk $\left(^{*}\right)$. A star tree is a prefix tree where each level represents an attribute and each node contains a partition value, an 
aggregate value, and pointers to the first child and the next sibling. Any node denoted by an asterisk $\left({ }^{*}\right)$ is a star node, and can be pruned, because it does not satisfy the aggregation condition. According to Apriori-like pruning, any partition that contains any star node can be pruned. To compute the iceberg cube query shown in Figure 2.5, StarCubing needs to construct the star tree and star table shown in Figure 2.11. According to Table 2.8, the single-attribute partitions Don, Thomas, DVD, Stereo, and Jan, do not meet the minimum support threshold of 2 , so they are added to the star table. The star tree shown in Figure 2.12 is a compressed representation of the database table Sales shown in Table 2.1 with the single-attribute partitions in the star table collapsed to star nodes. The star node Salesperson* with a count of 2 represents two single-attribute partitions: Don and Thomas.

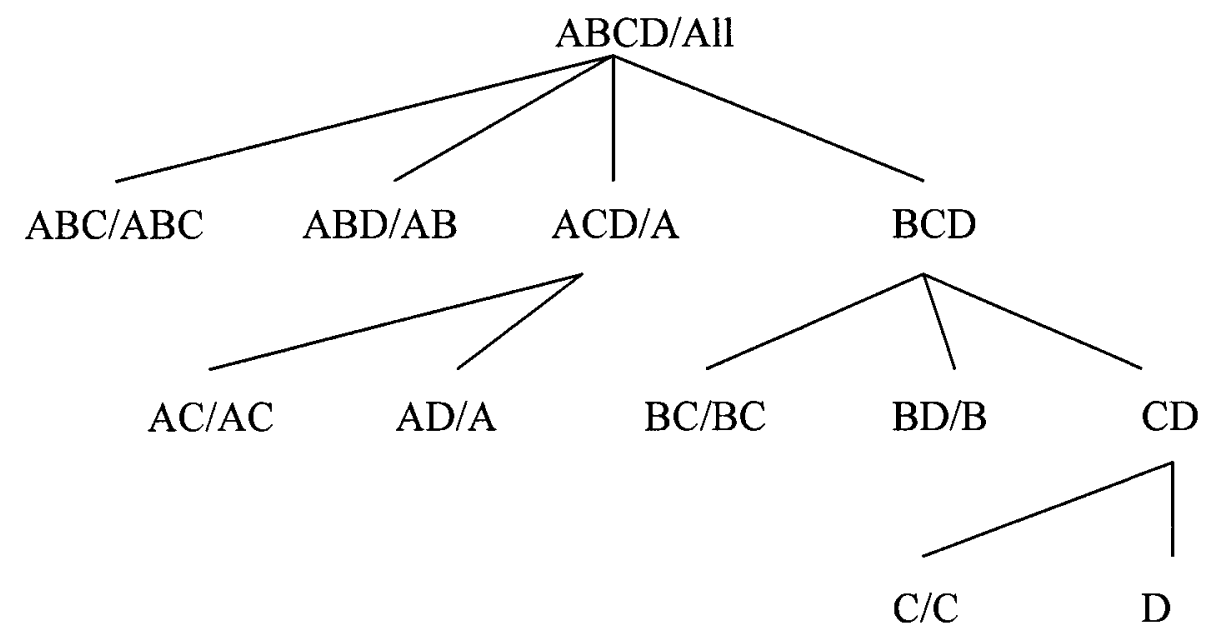

Figure 2.11: Star-Cubing Processing Tree

Table 2.8: One-Dimensional Partitions

\begin{tabular}{|l|l|l|}
\hline Attribute & Count $<$ MinSup & Count $>=$ MinSup \\
\hline Salesperson & Don, Thomas & Jane, Mike \\
\hline Product & DVD, Stereo & TV, VCR \\
\hline Month & Jan & Feb, Mar \\
\hline
\end{tabular}




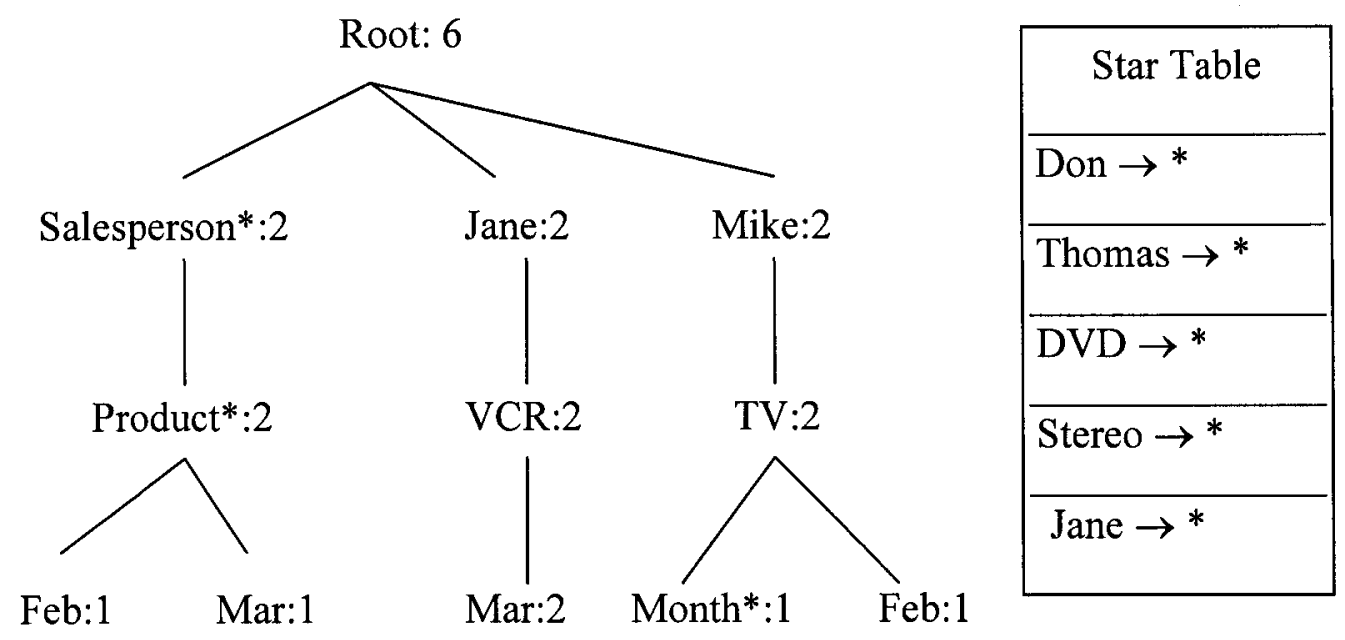

Figure 2.12: An Example of a Star Table and a Star Tree

Star-Cubing constructs child star trees from a parent star tree. For example, the star tree shown in Figure 2.12 can be used to construct three child star trees: SalespersonProduct, Salesperson-Month and Product-Month. The star tree Product-Month can be used recursively to construct two child star trees: Product and Month. Star-Cubing traverses a star tree in a depth-first fashion to determine output and applies Apriori-like pruning. Every star tree has its own star table, which needs to be dynamically constructed. According to the experiments conducted by Xin et al., Star-cubing outperforms the Multi-Way Array algorithm and BUC in most cases for sparse databases. It also outperforms the H-cubing algorithm, which is described in Section 2.3.6. For dense databases, the performance of Star-Cubing is about the same as the Multi-Way Array algorithm, but faster than that of the other algorithms. The main limitation of Star-Cubing is that the star trees and star tables may be too large to fit into memory. Thus, the StarCubing algorithm is memory intensive. 


\subsubsection{Algorithms for Iceberg Cubes with Complex Measures}

The four algorithms that have been reviewed in subsections 2.3 .2 to 2.3 .5 only deal with iceberg cubes with simple measures, such as Count, Sum, Minimum, and Maximum. These measures are anti-monotonic, so they can be used for Apriori-like pruning. The Top-k $\boldsymbol{H}$-Cubing algorithm [Han et al., 2001] and the $\boldsymbol{T o p} \boldsymbol{N}-\boldsymbol{A} \boldsymbol{V} \boldsymbol{G}-\boldsymbol{M i n e r}$ algorithm [Chou and Zhang 2002] were proposed to solve a related but different problem, computing iceberg cubes with complex measures, such as Average. Average is not antimonotonic, so it cannot be used as the basis for Apriori-like pruning. Top- $k$ H-Cubing uses a weaker but anti-monotonic function, Top- $\boldsymbol{k}$ Average, as the measure. Top $N$-AVGMiner uses a different weaker but anti-monotonic function, Upper Bound Average (UBAverage), as the measure. Both these measures permit Apriori-like pruning.

The Top- $k \mathrm{H}$-Cubing algorithm is based on a hyper-tree structure called an $\boldsymbol{H}$-tree. Assuming that the minimum support is $k$ tuples, the Top-k Average function is defined as the average of the top $k$ tuples of a partition, which are the $k$ tuples with the largest counts. To use the Top- $k$ Average as a pruning function, each partition needs to have at least $k$ tuples, which corresponds to the minimum support threshold. The Top- $k$ Average is always at least as great as the Average (Mean). The Top- $k$ Average is calculated by a complex binning technique, and its accuracy depends on the value of $k$.

The TopN-AVG-Miner algorithm is based on a hyper-tree structure called a large group tree (LG-tree), which is similar to H-tree. The $\boldsymbol{U B}$-Average of a group-by is the largest average among the averages of its child groups-by. The UB-Average enables a large average in a child group-by to be passed to its parent group-by. 
The Top- $k$ H-Cubing and TopN-AVG-Miner algorithms are both memory intensive, because they store the whole database table in an H-tree and an LG-tree, respectively.

\subsubsection{Comparison of the Iceberg Cube Algorithms}

We summarize the four principal algorithms reviewed in this subsection in Table 2.9. We do not include the Top- $k$ H-Cubing and TopN-AVG-Miner algorithms, because they are a specialized version of the iceberg cube problem and cannot be adapted to the general iceberg cube problem.

Table 2.9: Summary of Four Iceberg Cube Algorithms

\begin{tabular}{|l|l|l|l|}
\hline Algorithm & $\begin{array}{l}\text { Shared } \\
\text { Computation }\end{array}$ & Apriori-like Pruning & Memory Intensive \\
\hline TDC & Yes & Weak & Yes \\
\hline Multi-Way Array & Yes & No & Yes \\
\hline BUC & No & Yes & No \\
\hline Star-Cubing & Yes & Yes & Yes \\
\hline
\end{tabular}

TDC and the Multi-Way Array algorithm are top-down approaches, and their principal advantage is shared computation and their principal disadvantage is no or weak pruning. BUC applies Apriori-like pruning to speed up computation, but it does not share computed results between groups-by. Star-Cubing features both shared computation and Apriori-like pruning, but it may run out of memory. 


\section{CHAPTER 3}

\section{MULTI-TREE CUBING}

A new algorithm called Multi-Tree Cubing that computes an iceberg cube from a database table is presented in Section 3.1. The Multi-Tree Cubing algorithm integrates top-down and bottom-up approaches, and therefore it features both shared computation and Apriori-like pruning. A comparison between the Multi-Tree Cubing algorithm and the other algorithms that were reviewed in Chapter 2 is given in Section 3.2.

\subsection{Approach}

According to the analysis in Section 2.3 , the key to creating an efficient iceberg cube algorithm is to leverage the commonality between parent and child groups-by. Since Apriori-like pruning and shared computation have been successfully exploited in the past, an algorithm that can do both may offer superior performance.

With the above considerations in mind, we designed a new algorithm called Multi-Tree Cubing (MTC), which is presented in Figure 3.1. Overall, Multi-Tree Cubing is a top-down approach, so it uses orderings to cover all possible groups-by. An ordering $O$ is said to cover a group-by $G$ if $G$ can be computed during the same pass over the data as group-by $O$, which is the group-by with the same name as the ordering. However, MTC's processing order is the opposite of TDC's. Figure 3.2 shows the processing tree for the Multi-Tree Cubing algorithm for four attributes. Unlike with any previous algorithm, ordering D is processed first and ordering $\mathrm{ABCD}$ is processed last with MTC. 


\subsubsection{The Multi-Tree Cubing Algorithm}

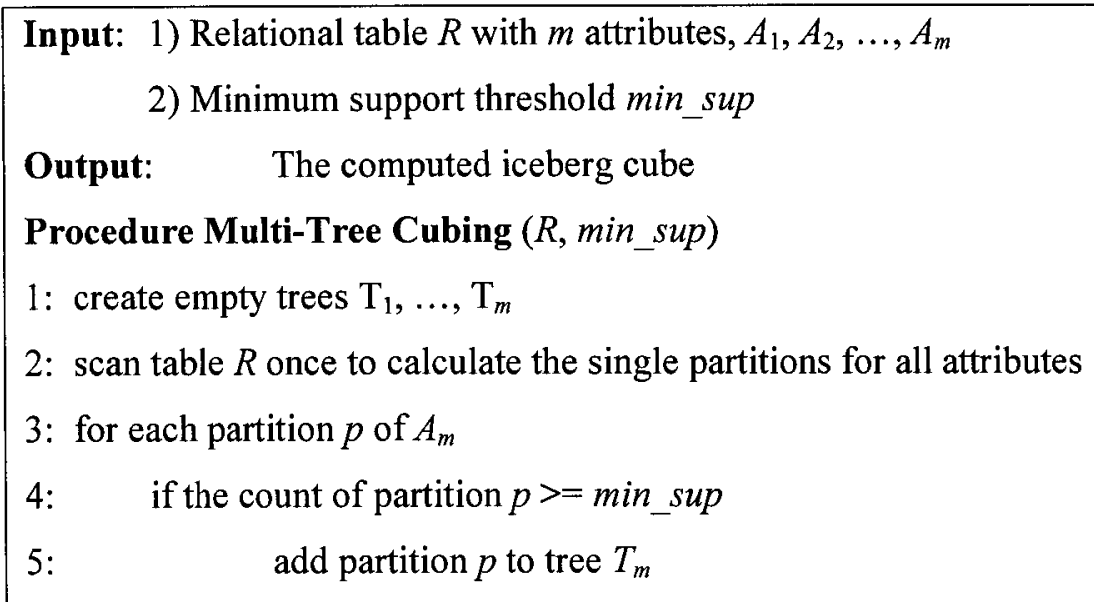

6: traverse tree $T_{m}$ to output results and add $T_{m}$ to the processed tree list $L_{t}$

7: for $i=m-1$ to 1 by -1

8: $\quad$ for each partition $p$ of $A_{i}$

9: $\quad$ if the count of partition $p>=$ min_sup

10: $\quad$ add partition $p$ to the first level of tree $T_{i}$

11: for each tuple of $R$

12: $\quad$ search tree $T_{i}$ for a match with the current tuple on attribute $A_{i}$

13: $\quad$ if a match is found

14: $\quad$ for each tree $T$ in $L_{l}$

15: $\quad$ search tree $T$ for matches with the current tuple on the prefix attributes

16: for each of the attribute matches

17: either add a new node $N$ with a count of 1 to tree $T_{i}$ or increment the count of the matched existing node

18: traverse tree $T_{i}$ depth first, for each node in the tree

19: $\quad$ if $\operatorname{count}(N)>=$ min_sup

20: $\quad$ output a result

21: $\quad$ else

22: $\quad$ prune the branch extending from the node $N$

23: $\quad$ add tree $T_{i}$ to the end of the processed tree list $L_{t}$

Figure 3.1: The Multi-Tree Cubing Algorithm 


\section{$\underline{8 A B C D}$}

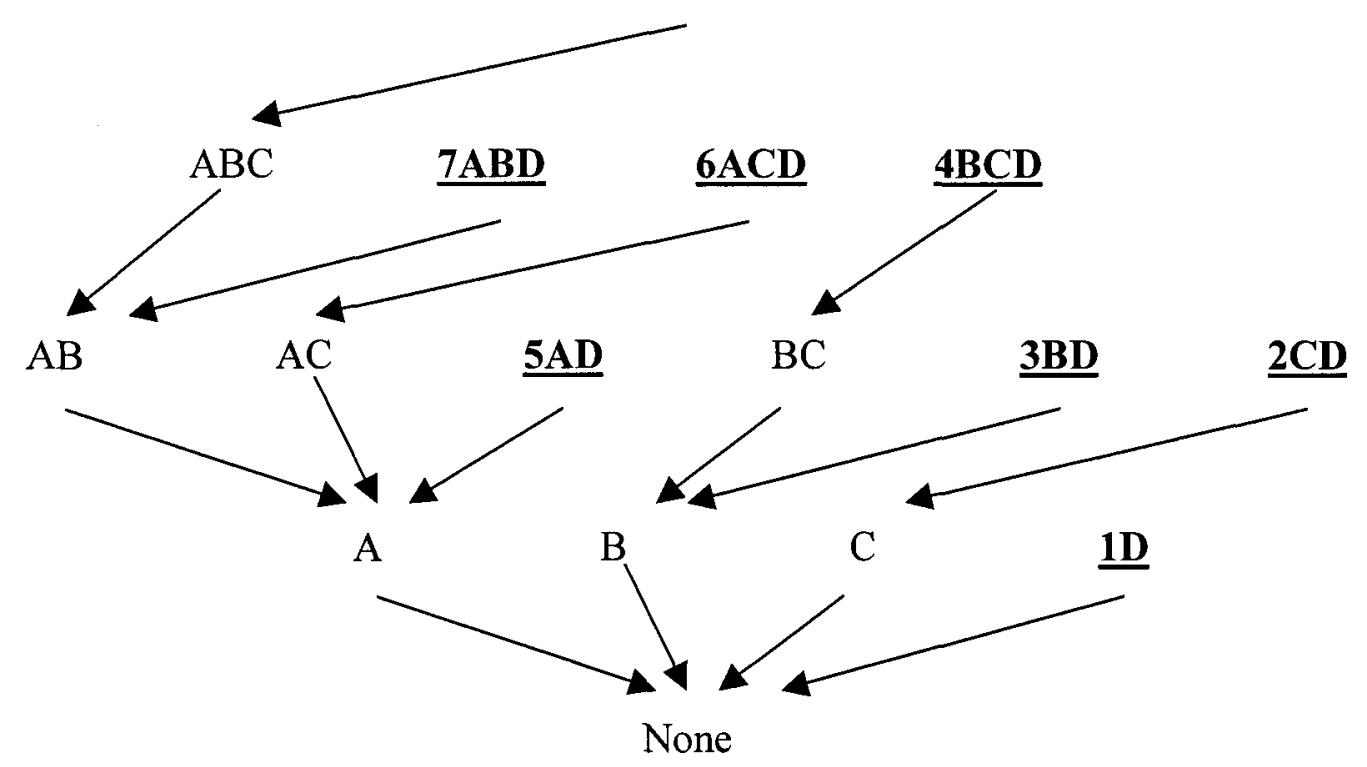

Figure 3.2: The Multi-Tree Cubing Processing Tree

An interesting observation concerning Figure 3.2 is that we can obtain the orderings for each attribute by adding it as prefix to the already processed orderings. For example, ordering $D$ is processed first. To get the orderings for attribute $C$, we add $C$ as a prefix to $D$ to obtain $C D$. Similarly, to get the orderings for attribute $B$, we add $B$ as a prefix to the already processed orderings $C D$ and $D$ to get $B C D$ and $B D$. This observation motivated the design of the more accurate pruning used in the Multi-Tree Cubing algorithm. First, all single partitions are computed. Then, $C D$ is computed from $C$ and $D$. Since the partitions of $C$ and $D$ that do not meet the minimum support condition have already been identified, any $C D$ partitions involving them can be safely pruned. As a result, fewer partitions are computed. 


\subsubsection{The Prefix Tree Data Structure}

On current computers, a $\mathrm{I} / \mathrm{O}$ access to disk requires an average seek time of approximately $8 \mathrm{~ms}$, while a memory access requires approximately $8 \mathrm{~ns}$, so scanning from the database is much slower than retrieving from a tree stored in main memory. However, memory space is typically much smaller than disk space. Algorithms like HCubing or Star-Cubing are memory intensive because they not only store a representation of the whole database in memory but also store other auxiliary data, such as lookup tables. Although they take less I/O time for smaller databases, they may run out of memory on larger databases. On the other hand, BUC and TDC are not memory intensive, but they take considerable I/O time. For example, TDC needs one scan of the table to process each ordering, so in total, it needs $2^{m-1}$ scans in the worst case, where $m$ is the number of attributes in the table.

To achieve a balance between memory usage and I/O time in the Multi-Tree Cubing algorithm, we employed a specialized form of a prefix tree, called an $\boldsymbol{A P}$ tree, for data storage. Figure 3.3 shows the AP tree structure and a sample AP tree $T$ for attribute Salesperson. The defining characteristic of an AP tree is the presence of two types of nodes: attribute nodes (A) and partition nodes (P). An attribute node stores an attribute name, the number of partitions (values for that attribute), and pointers to its partition nodes. Several attribute nodes can appear at the same level, and the same attribute can appear in attribute nodes at different levels. A partition node stores the partition value, count, and possibly pointers to attribute nodes. Attribute nodes and partition nodes occur at alternate levels in a tree. For example, the nodes shown in boldface in Figure 3.3 are attribute nodes, and the other nodes are partition nodes. An attribute node always points 
to at least one partition node, but a partition node may not point to any attribute node. In other words, an attribute node cannot be a leaf node, but a partition node can. The set of values at an attribute node or a partition node is arranged in lexicographical order, which facilitates binary search.

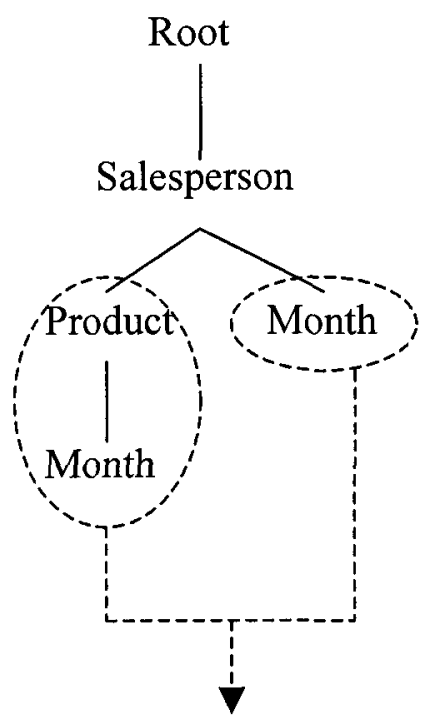

Sub-Trees

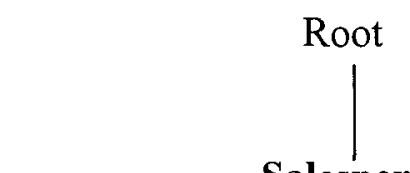

Salesperson

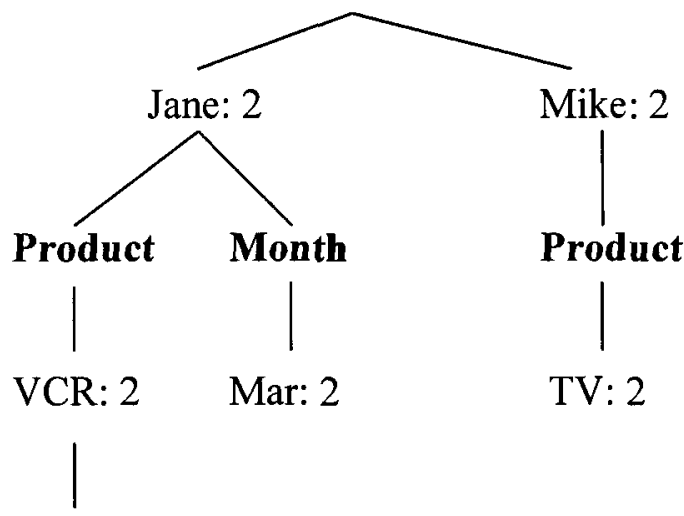

Month

Mar: 2

Figure 3.3: The AP Tree Structure and a Sample AP Tree $T$

The AP tree is similar to the H-tree, which was developed for the Top- $k \mathrm{H}$-Cubing algorithm, and the LG-tree, which was developed for the TopN-AVG-Miner algorithm. One difference is that both $\mathrm{H}$-trees and LG-trees include header tables and pointers from these header tables to tree nodes, while AP trees do not have header tables, which simplifies the Multi-Tree Cubing algorithm. A second difference is that AP trees have two distinguished types of nodes, namely attribute nodes and partition nodes, while neither of the other two algorithms has distinguished classes of nodes.

An AP tree contains all of the orderings for one attribute. For example, the sample tree in Figure 3.3 is for attribute Salesperson, which has two orderings: Salesperson- 
Product-Month and Salesperson-Month. The two orderings share the same prefix, which is the attribute Salesperson. The two sub-trees of the tree $T$ in Figure 3.3 are the trees for Product-Month and Month. Accordingly, we call $T$ the super-tree for the two sub-trees. The results from computing the sub-trees are used for the construction and pruning of their super-tree.

The nature of the AP tree determines that each AP tree can be used to compute multiple groups-by simultaneously. For example, the tree in Figure 3.3 can be used to compute three groups-by simultaneously: Salesperson-Product, Salesperson-ProductMonth, and Salesperson-Month. Group-by Salesperson is computed during the first table scan and used later for any possible pruning of the three groups-by.

\subsubsection{An Example of the Multi-Tree Cubing Algorithm}

We demonstrate the Multi-Tree Cubing algorithm by going through an example. Given the input table shown in Table 2.1, the Multi-Tree Cubing algorithm computes the iceberg cube corresponding to the iceberg cube query shown in Figure 2.5.

In line 1 of Figure 3.1, Multi-Tree Cubing creates the empty trees for all attributes. In line 2, MTC scans the input table once to calculate the single partitions for all of the attributes. For the last attribute $A_{m}$, which in this case is Month, only one ordering is possible, so only one prefix tree needs to be constructed. The results for attribute Month are shown in Figure 3.4(a). Partitions Feb and Mar satisfy the minimum support condition, so they are output as results and also added to the prefix tree (lines 3-6). One attribute node and two partition nodes for them are added to tree $T_{3}$, as shown in Figure 3.4(b). Then tree $T_{3}$ is added to the processed tree list $L_{t}$. Now, all of the partitions 
starting from the last attribute Month have been correctly computed and the results are shown in Table 3.1.

\begin{tabular}{|l|l|}
\hline Partition & Count \\
\hline Jan & 1 \\
\hline Feb & 2 \\
\hline Mar & 3 \\
\hline
\end{tabular}

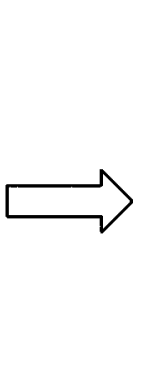

(a)

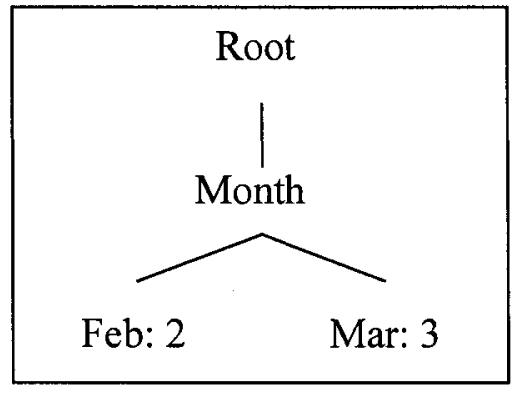

(b)

Figure 3.4: Single Partitions and Tree $T_{3}$ for Attribute Month

Table 3.1: Results for Attribute Month

\begin{tabular}{|l|l|l|}
\hline Group-by & Partition & Count \\
\hline Month & Feb & 2 \\
\hline Month & Mar & 3 \\
\hline
\end{tabular}

Next, on the first iteration of the loop in lines 7-23, MTC builds the first level of tree $T_{2}$ for current attribute $A_{i}$, which in this case is Product, as shown in Figure 3.5(b), based on its single partitions, as shown in Figure 3.5(a).

\begin{tabular}{|l|l|}
\hline Partition & Count \\
\hline VCR & 2 \\
\hline TV & 2 \\
\hline DVD & 1 \\
\hline Stereo & 1 \\
\hline
\end{tabular}

(a)

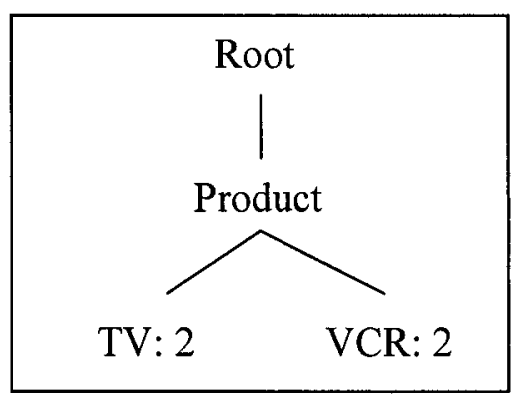

(b)

Figure 3.5: Single Partitions and Tree $T_{2}$ for Attribute Product

In line 11, MTC begins scanning the input table again by reading in the first tuple (Jane, $V C R, M a r)$. In line $12, V C R$ is found in tree $T_{2}$. So, according to lines 13-15, the only tree $T_{3}$ in the processed tree list $L_{t}$ is searched for Mar. Since a match is found, MTC 
adds an attribute node Month and a partition node Mar to tree $T_{2}$ and sets the count of node Mar to 1 (lines 16-17). Then lines 12-17 are repeated for the second tuple (Mike, TV, $J a n$ ), and trees $T_{2}$ and $T_{3}$ are searched for $T V$ and Jan, respectively. Although $T V$ is found in $T_{2}$, Jan is not found in $T_{3}$, so MTC does not add a node to $T_{2}$. The third tuple is identical to the first one, so $V C R$ is found in $T_{2}$ and Mar is found in $T_{3}$. Since attribute node Month and partition node Mar already exist in $T_{2}$, MTC does not add them again. Instead it increments the count of partition node Mar by 1 . After this process is repeated for the three remaining tuples, tree $T_{2}$ is as shown in Figure 3.6.

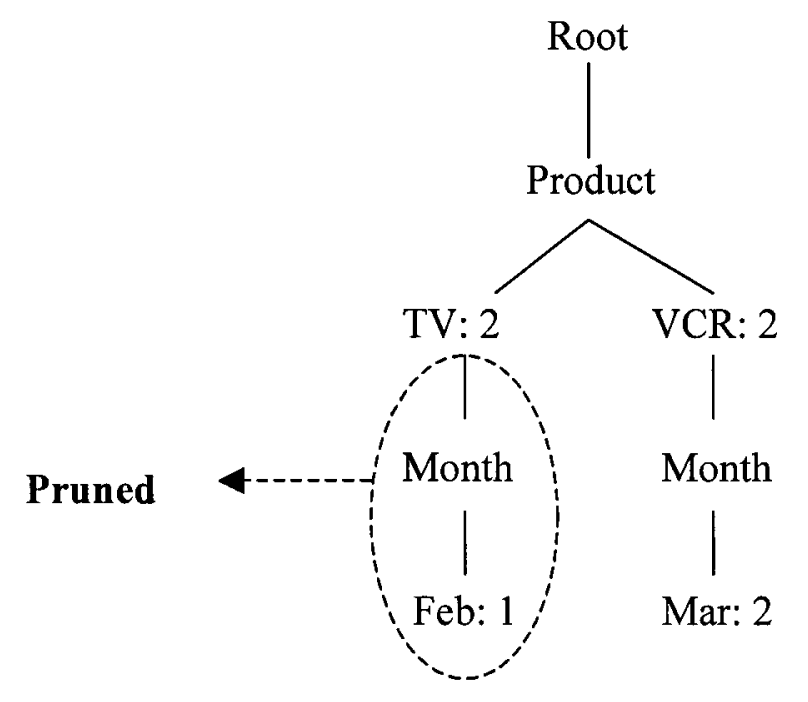

Figure 3.6: Tree $T_{2}$ for Attribute Product

Then, in lines 18-20, MTC begins traversing tree $T_{2}$ in a depth-first manner and outputs a result for node $T V$, because its count is greater than or equal to the threshold of 2. The next node is $F e b$ and its count is below 2, so it is pruned (lines 21-22). Feb does not have any siblings, so its parent, attribute node Month, is also pruned. MTC continues traversing the tree until all nodes in tree $T_{2}$ have been examined. Then, MTC adds tree $T_{2}$ to the processed tree list $L_{t}$, which now has two trees in it: $T_{2}$ and $T_{3}$. Now, all the 
partitions starting from the attribute Product have been correctly computed and the results are as shown in Table 3.2 .

Table 3.2: Results for Attribute Product

\begin{tabular}{|l|l|l|}
\hline Group-by & Partition & Count \\
\hline Product & TV & 2 \\
\hline Product & VCR & 2 \\
\hline Product-Month & VCR-Mar & 2 \\
\hline
\end{tabular}

In the second iteration of the loop in lines 7-23, MTC builds the first level of tree $T_{1}$ for attribute Salesperson based on its single partitions. It scans the input table for the third time. For each tuple, it searches the partitions of attribute Salesperson in tree $T_{1}$, searches the partitions of group-by Product-Month in tree $T_{2}$, and searches the partitions of attribute Month in tree $T_{3}$. A match in $T_{1}$ results in searching each tree in the processed tree list $L_{t}$. Every successful match of $T_{2}$ or $T_{3}$ results in either a new node being added to tree $T_{1}$ if it did not previously exist or an increment of the count for the matched attribute node. Figure 3.7 shows tree $T_{1}$ after all tuples have been processed.

MTC traverses tree $T_{1}$ to output results and prune the nodes that do not meet the minimum support condition. The results are shown in Table 3.3. At this point, all the partitions starting from the attribute Salesperson have been correctly computed and the Multi-Tree Cubing algorithm has completed the computation of the iceberg cube.

Table 3.3: Results for Attribute Salesperson

\begin{tabular}{|l|l|l|}
\hline Group-by & Partition & Count \\
\hline Salesperson & Jane & 2 \\
\hline Salesperson-Product & Jane-VCR & 2 \\
\hline Salesperson-Product-Month & Jane-VCR-Mar & 2 \\
\hline Salesperson & Mike & 2 \\
\hline Salesperson-Product & Mike-TV & 2 \\
\hline
\end{tabular}




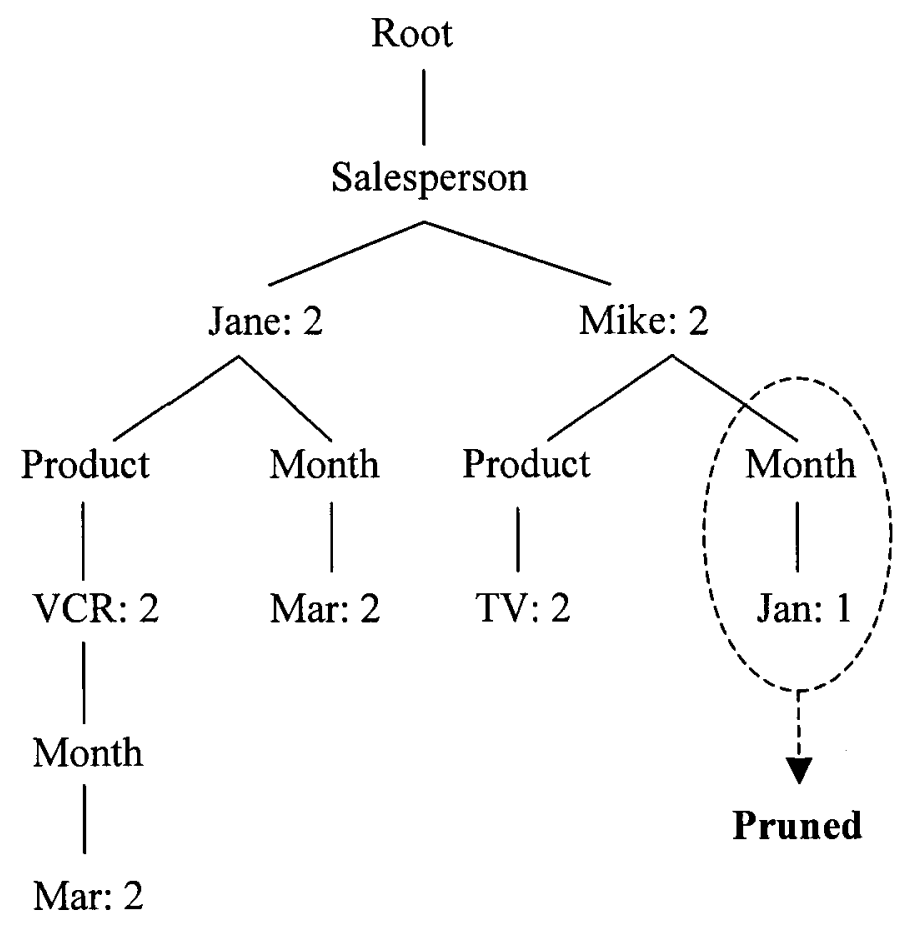

Figure 3.7: Tree $T_{1}$ for Attribute Salesperson

Table 3.4 shows all partitions that are processed, including the output ones that meet the minimum support condition and the pruned ones that do not.

\subsection{Comparison of Multi-Tree Cubing and Other Iceberg Cube Algorithms}

For a table with $m$ attributes $n$ tuples, and at most $k$ different values for each attribute, the Multi-Tree Cubing algorithm requires $m$ scans of a database containing $n$ records of size proportional to $k$, so MTC's I/O time is $O(m n k)$; MTC needs to build $m$ AP trees in the worst case and each AP tree can have at most $(k+1)^{m}$ nodes, which includes $(k+1)^{m-1}$ attribute nodes and $k(k+1)^{m-1}$ partition nodes. So, MTC's memory usage is $O\left(k^{m}\right)$. The I/O time for MTC is more than the one scan required by Star-Cubing but less than the $2^{m-1}$ scans required by TDC. The memory usage for MTC is more than that for BUC, which is a divide-and-conquer strategy, but less than that of Star-Cubing, which stores the whole input table. 
Table 3.4: Summary of MTC Computation Process

\begin{tabular}{|c|l|l|c|l|}
\hline Scan & Group-by & Partition & Count & Output/Pruned \\
\hline 1 & Month & Jan & 1 & Pruned \\
\hline 1 & Month & Feb & 2 & Output \\
\hline 1 & Month & Mar & 3 & Output \\
\hline 2 & Product & DVD & 1 & Pruned \\
\hline 2 & Product & Stereo & 1 & Pruned \\
\hline 2 & Product & TV & 2 & Output \\
\hline 2 & Product-Month & TV-Feb & 1 & Pruned \\
\hline 2 & Product & VCR & 2 & Output \\
\hline 2 & Product-Month & VCR-Mar & 2 & Output \\
\hline 3 & Salesperson & Thomas & 1 & Pruned \\
\hline 3 & Salesperson & Don & 1 & Pruned \\
\hline 3 & Salesperson & Jane & 2 & Output \\
\hline 3 & Salesperson-Product & Jane-VCR & 2 & Output \\
\hline 3 & Salesperson-Product-Month & Jane-VCR-Mar & 2 & Output \\
\hline 3 & Salesperson-Month & Jane-Mar & 2 & Output \\
\hline 3 & Salesperson & Mike & 2 & Output \\
\hline 3 & Salesperson-Product & Mike-TV & 2 & Output \\
\hline 3 & Salesperson-Month & Mike-Jan & 1 & Pruned \\
\hline
\end{tabular}

The major computation costs of the Multi-Tree algorithm are the AP tree construction and traversal operations, both of which are memory based. The tree construction includes the partition search and new node insertion. The partition search is a binary search that requires $O\left(\log _{2} k\right)$ comparisons for each level, where $k$ is the largest cardinality of attributes $A_{1}, A_{2}, \ldots, A_{m}$. So, for partition search, MTC needs the amount of time that is specified in formula 3.1 to search the tree built for attribute $\mathrm{A}_{i}$ :

$$
2^{m-i} * \log _{2} k
$$


where $i=1,2, \ldots, m$. Since a new node is added only if the partition search finds a match and locates where to add it, the time required for the new node insertion is much less than that of the partition search. So, MTC's computation time is $O\left(n^{*} \log _{2} k^{*} 2^{m-i}\right)$.

The Multi-Tree Cubing algorithm starts with the smallest tree and new trees may grow deeper and wider than the previous trees. However, each new tree is always based on the single partitions calculated at the beginning that satisfy the minimum support condition and their sub-trees, which are pruned after traversal. Thus, each new tree represents the minimum computation space that is required to compute all of the partitions for one attribute.

To summarize all algorithms discussed in this thesis, we add the Multi-Tree Cubing algorithm to the algorithm summary shown in Table 2.4 in Section 2.3.7 to obtain the new summary shown in Table 3.5.

Table 3.5: Summary of Five Iceberg Cube Algorithms

\begin{tabular}{|c|c|c|c|}
\hline Algorithm & $\begin{array}{c}\text { Shared } \\
\text { Computation }\end{array}$ & Apriori-like Pruning & Memory Intensive \\
\hline TDC & Yes & Weak & Yes \\
\hline Multi-Way Array & Yes & No & Yes \\
\hline BUC & No & Yes & No \\
\hline Star-Cubing & Yes & Yes & Yes \\
\hline Multi-Tree Cubing & Yes & Yes & No \\
\hline
\end{tabular}

From this table, we see that Multi-Tree Cubing and Star-Cubing feature both shared computation and Apriori-like pruning, while TDC, Multi-Way Array, and BUC have only one of these two features. However, Star-Cubing uses much more memory than Multi-Tree Cubing. 
Both Multi-Tree Cubing and TDC make use of orderings to achieve shared computation, but Multi-Tree Cubing processes multiple orderings during each scan of the input table, while TDC processes only one ordering during each scan. In addition, for each ordering, MTC has fewer partitions to process than TDC, because MTC applies Apriori-like pruning at the partition level, while TDC's pruning is at the attribute level. As a result, the shared computation of Multi-Tree Cubing is more efficient than that of TDC.

Both Multi-Tree Cubing and BUC apply Apriori-like pruning, but MTC's pruning is more accurate. For example, suppose that partition $b_{2} c_{2} d_{2}$ is in fact the only result for group-by $B C D$. MTC would process the $C D$ and $B$ groups-by before considering $B C D$. If MTC found that $c_{2} d_{2}$ is the only result for $C D$, and $b_{2}$ is the only result for $B$, it would not process the partitions of $B C D$ other than $b_{2} c_{2} d_{2}$. On the other hand, BUC would process the B groups-by before $B C D$, but it would not process $C D$ before $B C D$. Thus, if BUC found that $b_{2}$ is the only result for $B$, it would process all of the partitions of $B C D$ where $B$ is $b_{2}$. As a result, partitions such as $b_{2} c_{1} d_{2}$ or $b_{2} c_{3} d_{1}$ would be processed by BUC, but not by MTC. 


\section{CHAPTER 4}

\section{EXPERIMENTS AND RESULTS}

In this chapter, we give the results of an experimental evaluation of the MultiTree Cubing algorithm. First, in Section 4.1, we describe the details of our experimental design, which include algorithms to be compared, series of experiments to be performed, implementation platforms, and other technical specifications. Then, in Section 4.2, we report the experimental results by providing a series of charts along with explanations. Finally, in Section 4.3, we summarize the findings from these experiments.

\subsection{Experimental Design}

We implemented the Multi-Tree Cubing algorithm using the Java 1.18 programming language, the Open Database Connect (ODBC) protocol, and the Microsoft Access 2000 database system. We also implemented the BUC algorithm using the same software platforms. To evaluate the performance of the Multi-Tree Cubing algorithm, we designed the following five series of experiments.

1. Table Size

2. Minimum Support

3. Cardinality

4. Dimension (Number of Attributes)

5. Data Skew

In each series, we compared the performance of Multi-Tree Cubing to that of BUC, which is a well-known iceberg cube algorithm. Of the four iceberg cube algorithms 
(See Table 2.9), only BUC is not memory intensive. Since Multi-Tree Cubing is also not memory intensive, BUC was the appropriate algorithm for comparison. All five series of tests were executed on a dedicated 598MHz Pentium III PC with $256 \mathrm{MB}$ of RAM. The time recorded is the elapsed time, which includes both computation time and I/O time. For each run with the same input parameter values, six times were recorded and the average of the middle four was calculated and used for creating the charts shown in Section 4.3. On the computer platform used for the experiments, separate information about the computation time or the I/O time was not available.

For our comparison algorithm, we chose BUC, because it performs more efficiently than TDC in most cases [Hamilton and Findlater 2003], and unlike StarCubing or H-Cubing, it is not memory intensive. According to [Beyer and Ramakrishnan 1999], the CountingSort method of BUC requires the input to be packed, which means that the cardinality must be known in advance and the values translated into integers from 0 to one less than its cardinality. If the input is not packed, then it can be packed by reading the input table once and creating a hashed symbol table. We followed their suggestion and implemented a pre-processing step to pack the input table before the actual BUC algorithm was started. The runtime of the pre-processing step was not included in the elapsed time reported for our experiments. We also implemented a postprocessing step that converts the integers in the cube results back to their original values. Similarly, the runtime of the post-processing step was not included in the elapsed time.

We performed extensive experiments with both real and synthetic databases. The Student database, which is a real database, was extracted from the University of Regina's student record system with student names removed. The full table contains 59,689 tuples 
in total. To obtain various sizes of Student tables, we used initial subsets of the full Student table. For example, the Student 20000 table was created by picking the first 20,000 records from the full Student table. The synthetic PSCS tables were generated by our data generator, which is detailed in Section 4.2. All of the Student and PSCS databases used in our experiments are listed in Table 4.1. The meaning of the Zipf values shown in the table is explained in Section 4.2.

The performance of the Multi-Tree Cubing algorithm is sensitive to the order of the attributes. For the five experiments we conducted, the attributes were arranged in cardinality descending order, which gives superior performance. For any other order, the trees commonly grow wider and increase the amount of memory required, possibly exhausting available main memory in the computer.

\subsection{The Synthetic Data Generator}

We developed a synthetic data generator in Visual Basic 6 to generate tables with different sizes, attributes with different cardinalities, data with different skew, etc. The data generator can create MS Access database tables with four attributes: Part, Supplier, Customer, and Store. The user interface of the data generator is shown in Figure 4.1.

Users can specify the number of tuples for the new table and a different cardinality for each attribute. The values of each attribute are integers between 0 and one less than the cardinality, which is the packed format required by the CountingSort method of the BUC algorithm. Therefore, BUC does not need pre-processing and post-processing steps when using the PSCS tables as input. Users can also set an individual Zipf value for each attribute. 
Table 4.1: Input Tables Used in Our Experiments

\begin{tabular}{|c|c|c|c|c|c|}
\hline Table Name & Type & Table Size & Dimension & $\begin{array}{l}\text { Zipf } \\
\text { (Skew) }\end{array}$ & Test Series \\
\hline Student 10000 & Real & 10,000 & 4 & & Table Size \\
\hline Student 20000 & Real & 20,000 & 4 & & Table Size \& Min Sup \\
\hline Student 30000 & Real & 30,000 & 4 & & Table Size \\
\hline Student 40000 & Real & 40,000 & 4 & & Table Size \\
\hline Student 50000 & Real & 50,000 & 4 & & Table Size \\
\hline Student & Real & 59,689 & 4 & & Table Size \& Min Sup \\
\hline Student33 & Real & 30,000 & 7 & & Dimension \\
\hline PSCS $20000 Z 0$ & Synthetic & 20,000 & 4 & 0 & Skew \\
\hline PSCS20000Z1 & Synthetic & 20,000 & 4 & 1 & Skew \\
\hline PSCS20000Z2 & Synthetic & 20,000 & 4 & 2 & Skew \\
\hline PSCS20000Z3 & Synthetic & 20,000 & 4 & 3 & Skew \\
\hline PSCS40000Z0 & Synthetic & 40,000 & 4 & 0 & Skew \\
\hline PSCS40000Z1 & Synthetic & 40,000 & 4 & 1 & Skew \\
\hline PSCS40000Z2 & Synthetic & 40,000 & 4 & 2 & Skew \\
\hline PSCS40000Z3 & Synthetic & 40,000 & 4 & 3 & Skew \\
\hline PSCS5000Z2 & Synthetic & 5,000 & 4 & 2 & Table Size \\
\hline PSCS10000Z2 & Synthetic & 10,000 & 4 & 2 & Table Size \\
\hline PSCS20000Z2 & Synthetic & 20,000 & 4 & 2 & Table Size \\
\hline PSCS30000Z2 & Synthetic & 30,000 & 4 & 2 & Table Size \& Min Sup \\
\hline PSCS40000Z2 & Synthetic & 40,000 & 4 & 2 & Table Size \\
\hline PSCS $20000 \mathrm{C} 2$ & Synthetic & 20,000 & 4 & 0 & Cardinality \\
\hline PSCS20000C6 & Synthetic & 20,000 & 4 & 0 & Cardinality \\
\hline PSCS20000C10 & Synthetic & 20,000 & 4 & 0 & Cardinality \\
\hline PSCS20000C14 & Synthetic & 20,000 & 4 & 0 & Cardinality \\
\hline PSCS $20000 \mathrm{C} 18$ & Synthetic & 20,000 & 4 & 0 & Cardinality \\
\hline PSCS $40000 \mathrm{C} 2$ & Synthetic & 40,000 & 4 & 0 & Cardinality \\
\hline PSCS $40000 \mathrm{C} 6$ & Synthetic & 40,000 & 4 & 0 & Cardinality \\
\hline PSCS40000C 10 & Synthetic & 40,000 & 4 & 0 & Cardinality \\
\hline PSCS $40000 \mathrm{C} 14$ & Synthetic & 40,000 & 4 & 0 & Cardinality \\
\hline PSCS40000C 18 & Synthetic & 40,000 & 4 & 0 & Cardinality \\
\hline
\end{tabular}




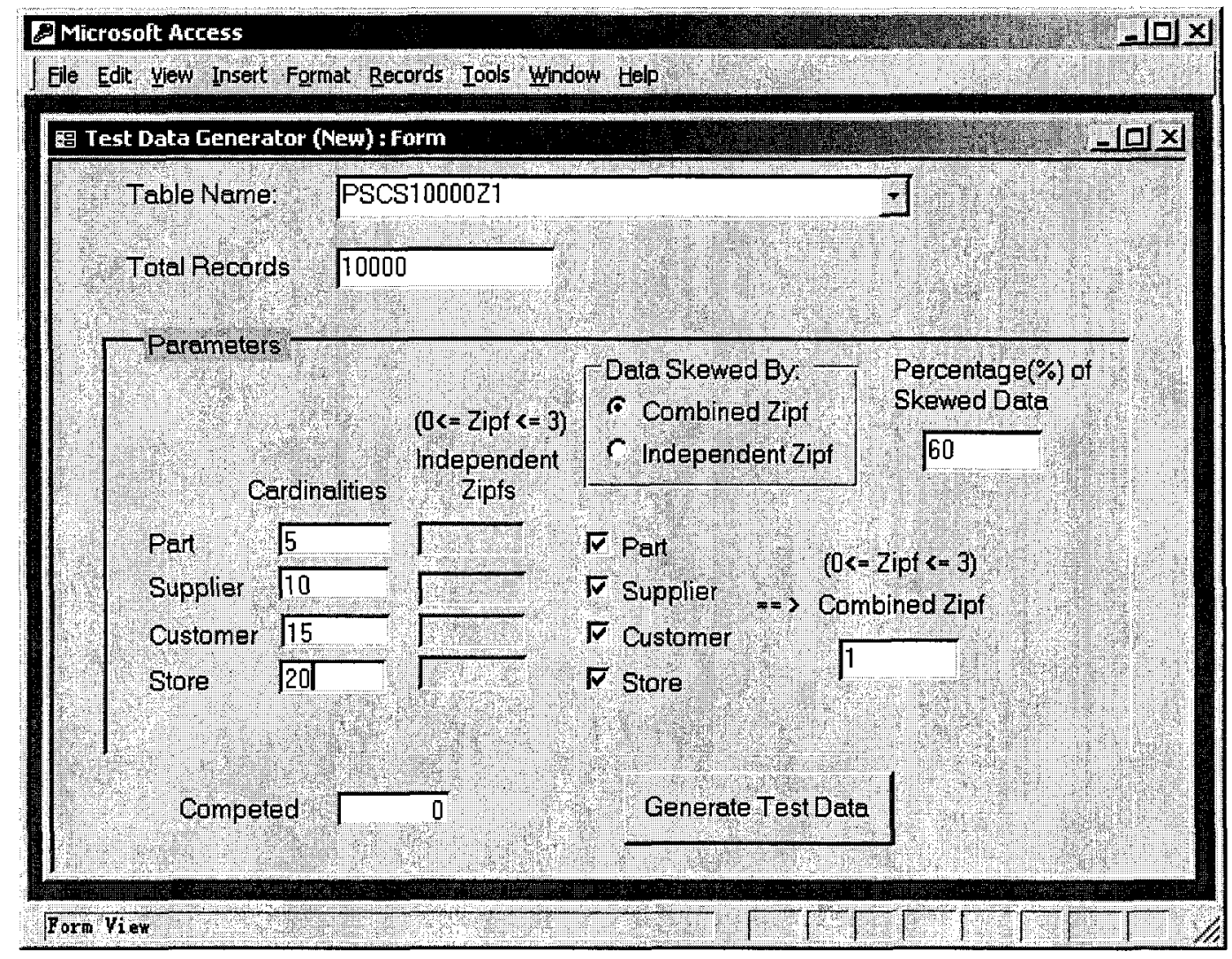

Figure 4.1: The User Interface of the Data Generator

The Zipf value is an integer between 0 and 3. When the Zipf value is 0 , the data is approximately evenly distributed, i.e., it is randomly drawn from a uniform distribution. When the Zipf value is greater than 0 , the data is skewed. The greater the Zipf value, the more skewed the data. A combined Zipf value can also be set for two or more attributes. In this case, these attributes are considered jointly and their combinations are skewed instead of skewing the values of a single attribute. In addition, when the combined Zipf value is set, a percentage parameter can be used to limit the number of tuples to be skewed. For example, if the combined Zipf value is set to 1 and the percentage of skewed data is set to $60 \%$, then $60 \%$ of the total tuples will be created using a Zipf value of 1 and the remaining $40 \%$ will have a uniform random distribution using a Zipf value of 0 . We 
create this type of test data, because it mimics databases encountered in practice, which may contain both roughly uniform data and skewed data. The design of the data generator is quite straightforward, except for the data skew. So here we only give more details about the data skew.

Equation (1) [Poosala 1995] defines the size-frequency relation:

$$
f(r)=A r^{-(1+\alpha)}, r=1,2, \ldots
$$

where $\alpha>=0$, and

$$
A=\sum_{r=1}^{\infty} r^{-(1+\alpha)}
$$

Based on Equation (1) and (2), we calculate the number of tuples for each value $r$ of an attribute. Although Equation (2) is a sum from 1 to $\infty$, in the implementation the summation loop was repeated from the value with most records to the value with the least records until either the last value or the number of records for a value was 1 . As a result, the implementation truncated the infinite series so that the summation of the number of tuples for all values equals to the table size, which is specified by the user. The $\alpha$ in Equations (1) and (2) is the Zipf parameter for the data generator. When $\alpha$ is 0 , the distribution is uniform and as $\alpha$ increases, the skew of the function increases. We performed experiments using the data generator to generate tables with various Zipf values, and the data distribution of these tables is shown in Figures 4.2-4.5.

Figure 4.2 shows the data distribution with Zipf values from 0 to 3. When the Zipf value is 0 , approximately 100 tuples were generated for each value in $[1,2, \ldots, 10]$, 


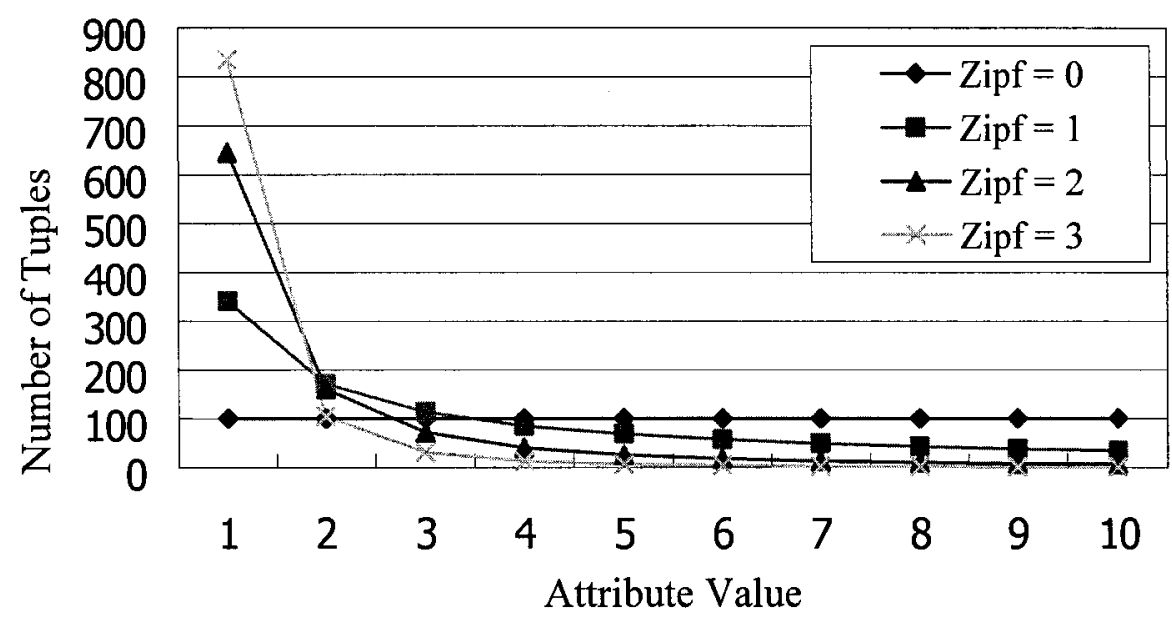

Figure 4.2: Data Distribution with Table Size $=1,000$ and Cardinality $=10$ so the data is evenly distributed. As the Zipf value was increased, the number of tuples generated for smaller values, such as 1 , also increased, while the number of tuples generated for larger values was decreased. So the data skew increased.

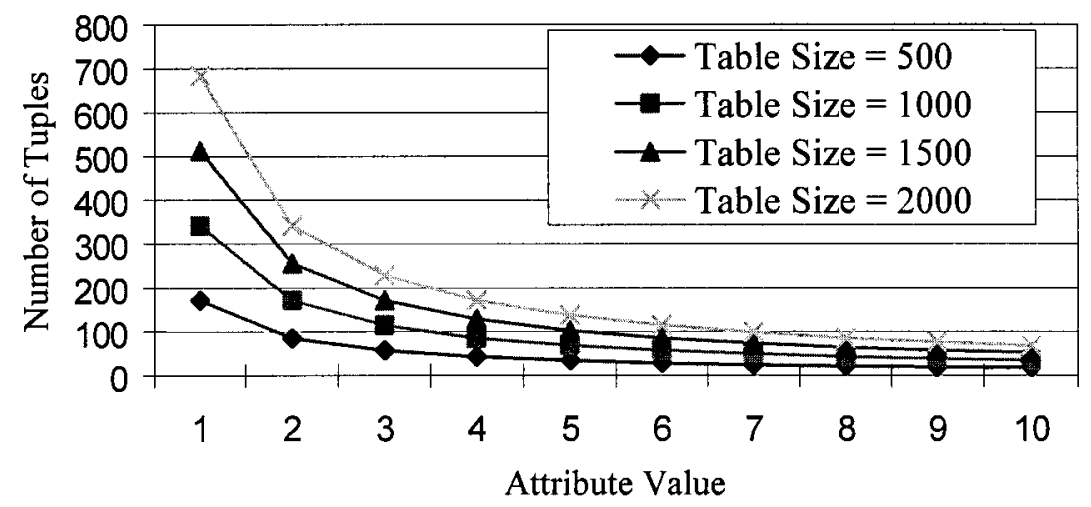

Figure 4.3: Data Distribution with Zipf $=1$ and Cardinality $=10$

In Figure 4.3, we can see that for the given Zipf value of 1, the four tables have different tables sizes, but the trends in their data distribution are very similar to each other. 


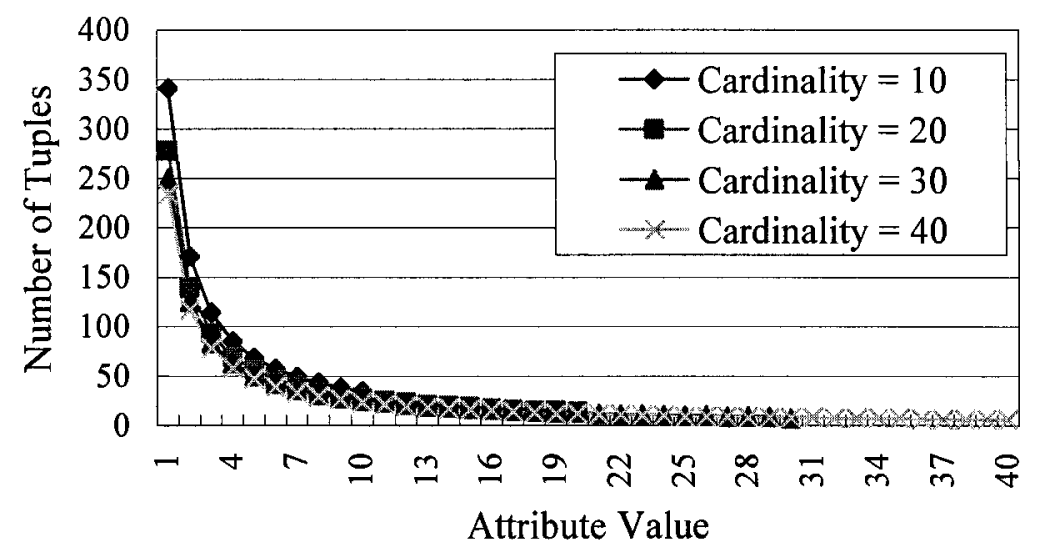

Figure 4.4: Data Distribution with Zipf $=1$ and Table Size $=1,000$

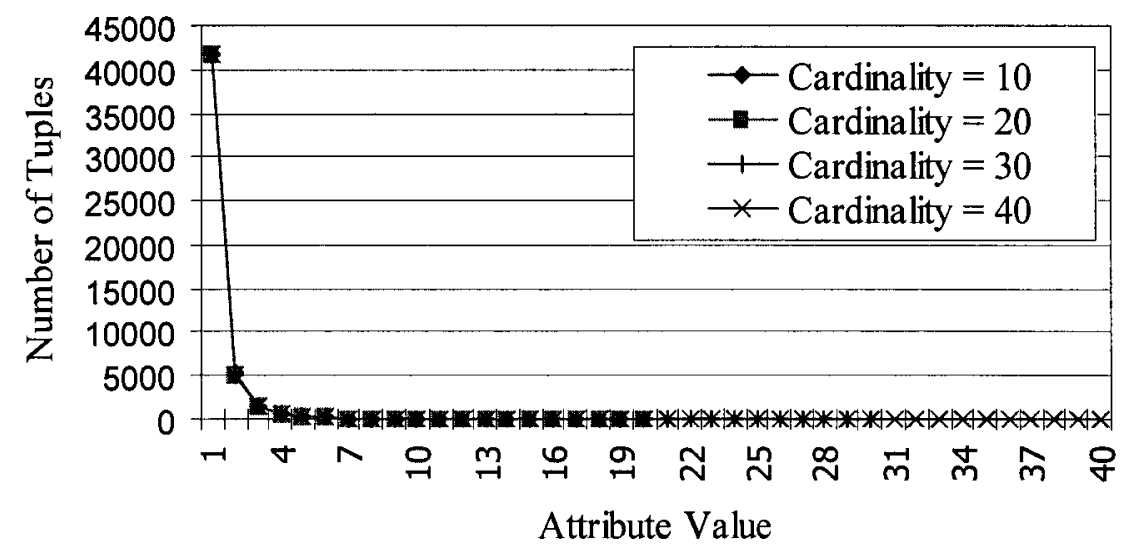

Figure 4.5: Data Distribution with Zipf $=3$ and Table Size $=50,000$

Figures 4.4 and 4.5 show that the data distributions for different cardinalities are almost identical. Furthermore, the data distribution in Figure 4.5 is highly skewed as the Zipf value was set to 3 . Other experiments (not shown) indicated that Zipf values higher than 3 give similar distributions to those shown in Figure 4.5. Therefore, we performed the remainder of our experiments using the range [0,3] for the Zipf parameter. From the 
results illustrated in Figures 4.2 to 4.5, we concluded that the data generator was working as we expected with respect to data skew.

\subsection{Experimental Results}

In this section, we describe the results for the five series of experiments comparing the performance of MTC and BUC.

\section{Series 1: Table Size}

Figures 4.6 and 4.7 show the performance of MTC and BUC for different sizes of Student tables, with the minimum support set to 1000 for Figure 4.6 and 2000 for Figure 4.7. Both algorithms responded linearly as the table size increases. This makes sense because the CountingSort, which is the major cost of BUC, is an $O(n)$ algorithm, and for MTC, the linear increase of the table size caused the linear increase of its $\mathrm{I} / \mathrm{O}$ time and computation time while the attributes and their cardinalities remain about the same. For smaller table sizes, the minimum support (1000) is relatively high in comparison to the table size and the running time of the two algorithms was about the same. For larger table sizes, the minimum support is relatively low and more partitions and tuples need to be processed, so both algorithms ran longer. The pruning in MTC pruning is more accurate than that in BUC, so MTC saved time by processing fewer partitions, as shown in Figure 4.6. For all of the various sizes of PSCS tables shown in Figure 4.8, MTC had a slightly better performance than BUC, which is consistent with the previous two charts. 


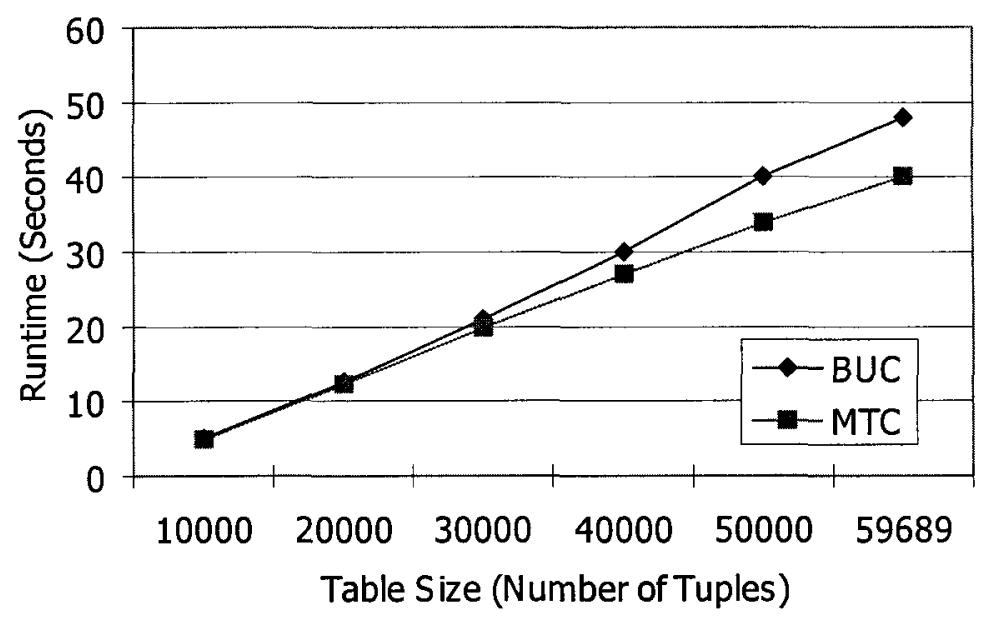

Figure 4.6: Table Size $($ Table $=$ Student, Dimension $=4, \operatorname{MinSup}=1,000)$

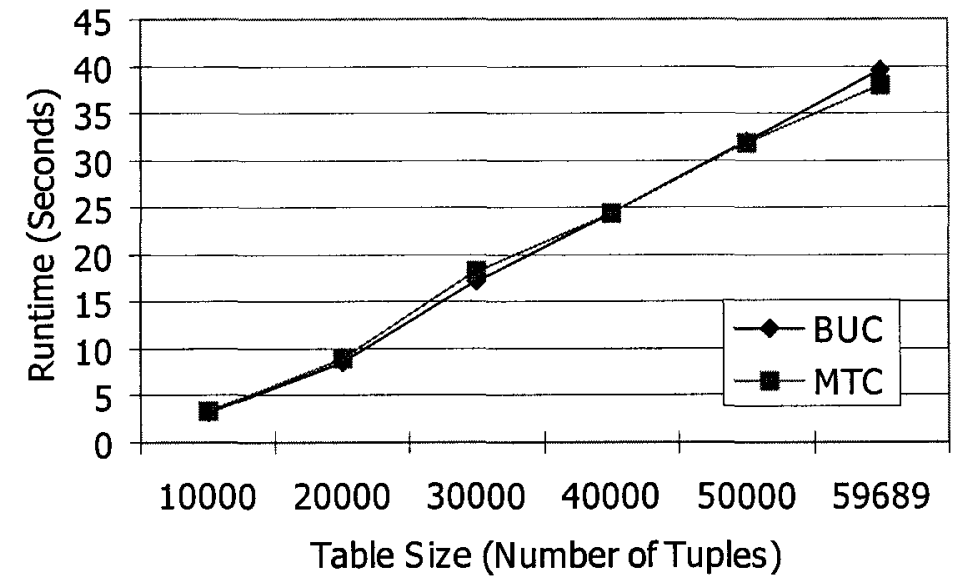

Figure 4.7: Table Size $($ Table $=$ Student, Dimension $=4$, MinSup $=2,000)$ 


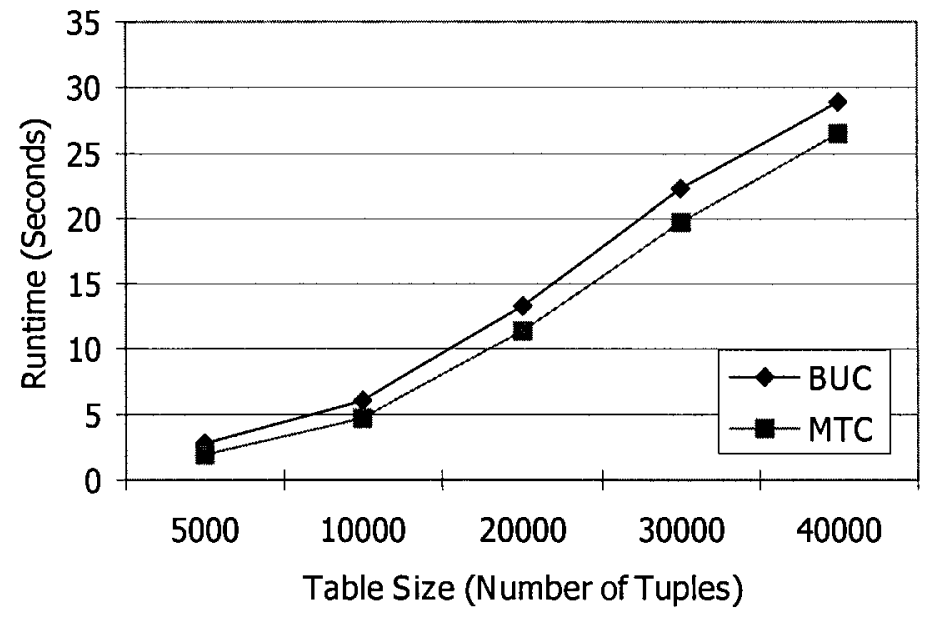

Figure 4.8: Table Size $($ Table $=$ PSCS, Dimension $=4$, MinSup $=500$, Zipf $=2)$

\section{Series 2: Minimum Support}

We used the Student table with 20,000 tuples for the tests shown in Figure 4.9 and the full Student table with 59,689 tuples for the tests shown in Figure 4.10. From both charts, we can see that when the minimum support increases, the runtime for BUC decreases more dramatically than that for MTC. MTC consistently performed better than BUC. The runtime of the two algorithms decreases when the higher minimum support enables them to achieve better pruning and process fewer partitions. At lower values for the minimum support threshold, there were more distinct partitions that met the minimum support, but the more accurate pruning in MTC enabled it to process fewer partitions than BUC. The ability of MTC to perform shared computation also contributed to its faster computation. For these two reasons, the runtime of MTC was approximately $50 \%$ of the runtime of BUC when the minimum support was set to 10, as shown in Figure 4.9.

As shown in Figure 4.11, MTC also performed better than BUC on synthetic data for various values of the minimum support threshold, which is consistent with its 
performance on the Student data. For all other series of experiments, minimum support values were selected corresponding to $5 \%$ or less of records in table. Such support values ensures that the algorithms were tested on the more difficult cases that might occur in practice.

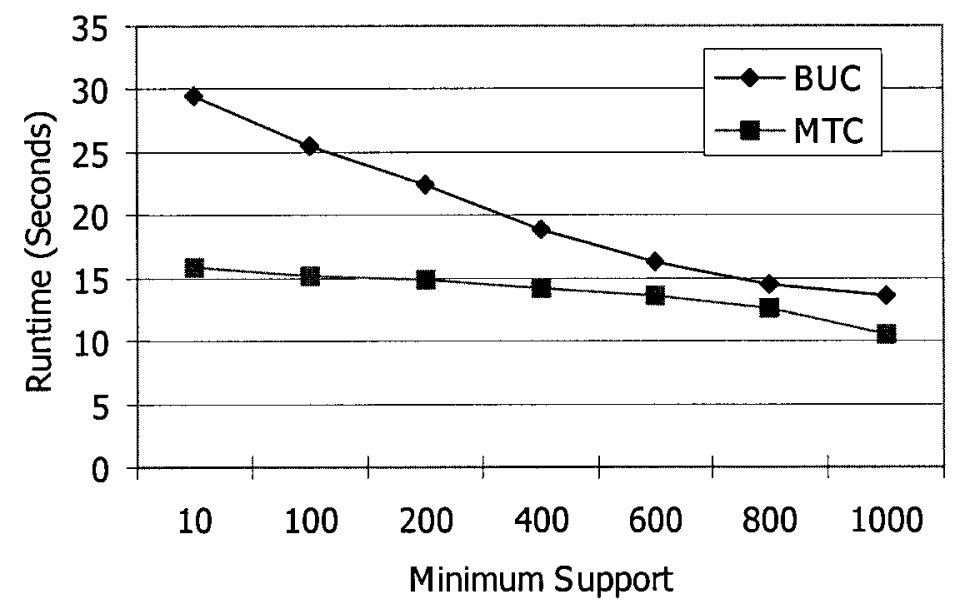

Figure 4.9: Minimum Support $($ Table $=$ Student, Size $=20,000$, Dimension $=4)$

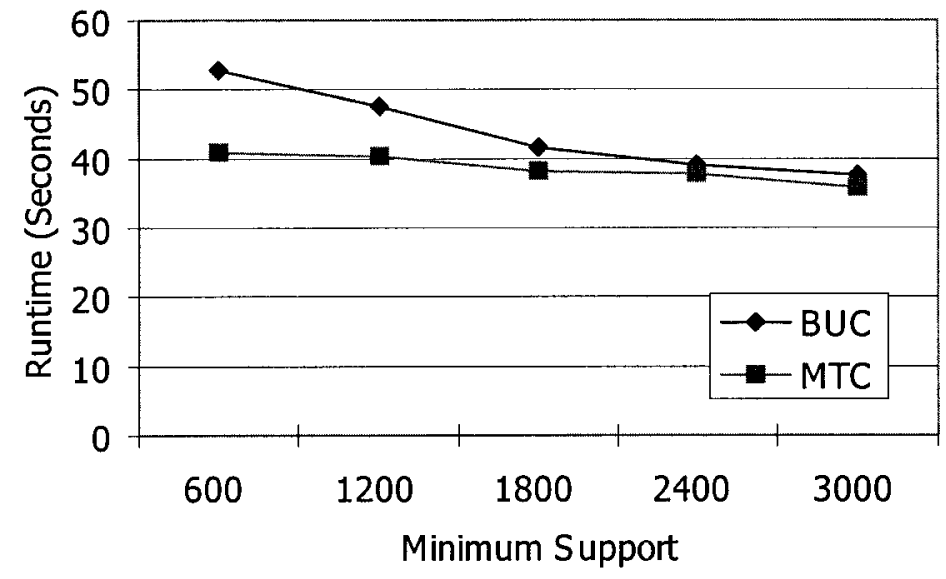

Figure 4.10: Minimum Support $($ Table $=$ Student, Size $=59,689$, Dimension $=4)$ 


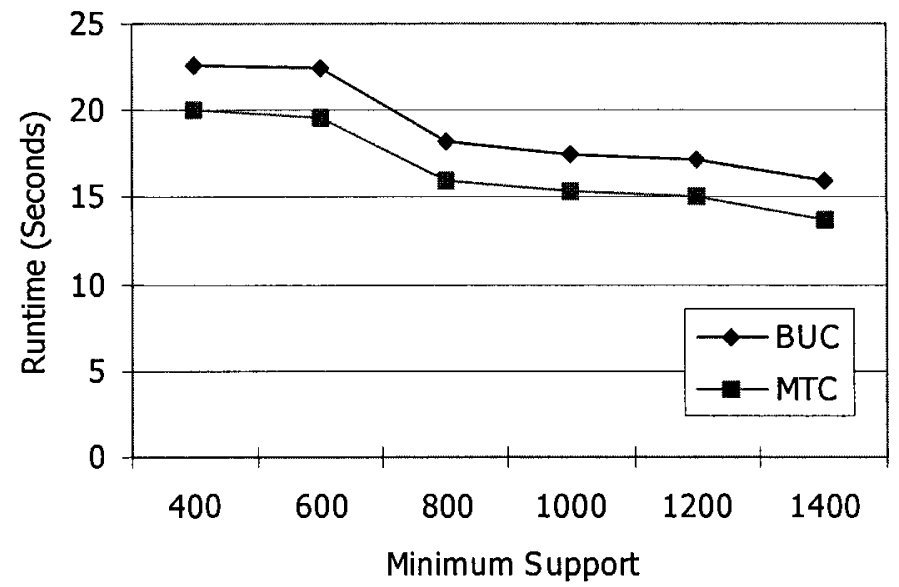

Figure 4.11: Minimum Support $($ Table $=$ PSCS, Size $=30,000$, Zipf $=2)$

\section{Series 3: Cardinality}

The full Student table has 21 attributes, but many attributes are not suitable for the data cube operation. For example, the Nation, Province and City attributes are not suitable because data values are missing for some students. The Pidm attribute, which represents the student number, is also not suitable. It is very useful for querying a specific student's information, but it is not a good choice for aggregations, such as the iceberg cube, because the value is unique to each tuple. Therefore, we chose seven attributes from the full Student table for the tests shown in Figure 4.12 and used four attributes for each run. The three attributes with the lowest cardinalities were included for all runs and a different attribute from the remaining four attributes was used for each run. The cardinality value shown in the chart is the cardinality of the varying attribute. For the two tests with the PSCS tables that are shown in Figure 4.13 and 4.14, we created ten tables, five for each test. For each run, the cardinalities of two of the attributes were fixed at 5 
and the cardinalities for the other two attributes were varied from 2 to 18 at an interval of 4.

As shown in Figure 4.12, 4.13 and 4.14, both MTC and BUC ran longer as the cardinality was increased. The combined effect of higher cardinality and low minimum support caused more distinct partitions to be needed, which takes longer to process. The more accurate pruning and the shared computation helped MTC to consistently outperform BUC. When the results among the three charts are examined, the advantage of MTC may seem to be more significant for the PSCS tables. Only one attribute was varied for the Student table, but two attributes were varied for the PSCS tables. Also, the Zipf value for the PSCS tables was 0, which corresponds to a uniform random distribution. The Zipf value for the Student table is not known, but it must be greater than 0 , because the data in this table were certainly not uniformly distributed. As will be described in more detail shortly with reference to the series of experiments concerning data skew (series 5), MTC appears to outperform BUC to the largest extent when the distribution is a uniform random distribution. Therefore, when the two factors, having only one varying attribute and having a higher Zipf value, occur together, we see a smaller difference in the performance of the two algorithms, such as in Figure 4.12, as opposed to cases where these two factors do not co-occur, such as in Figures 4.13 and 4.14. 


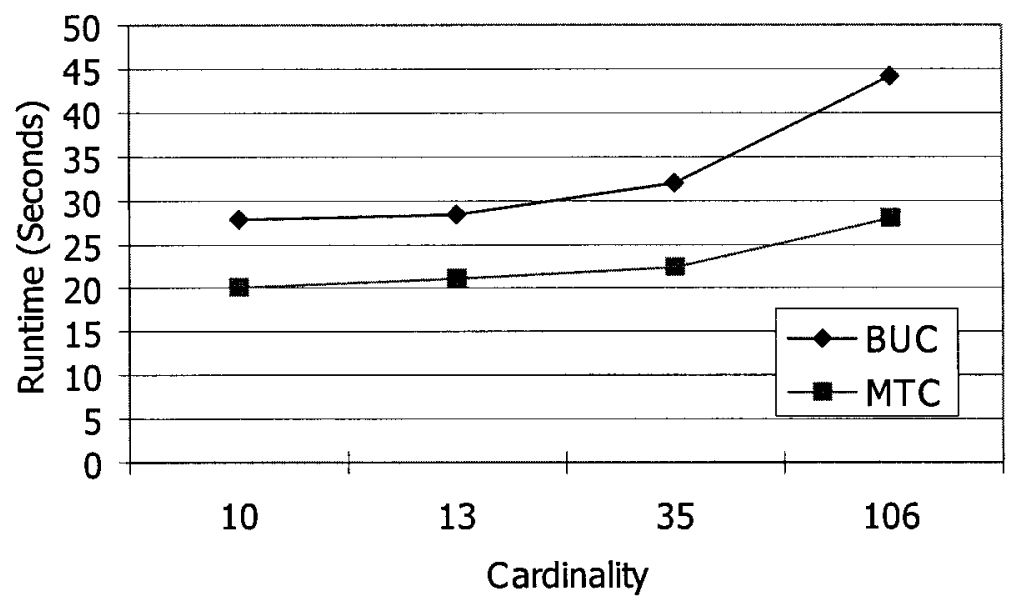

Figure 4.12: Cardinality $($ Table $=$ Student, Size $=30,000$, MinSup $=10)$

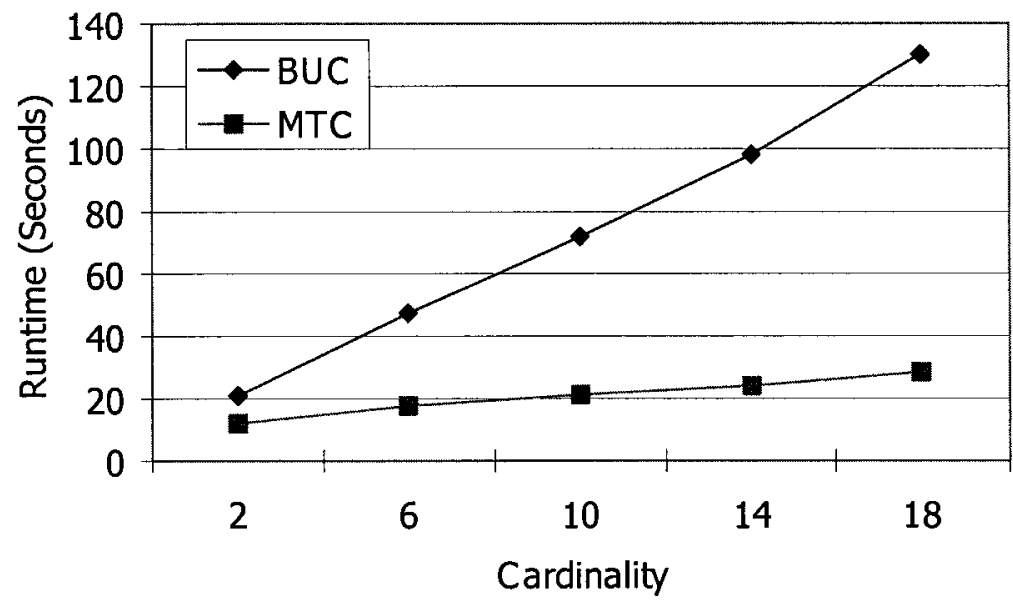

Figure 4.13: Cardinality $($ Table $=\operatorname{PSCS}$, Size $=20,000$, MinSup $=10$, Zipf $=0$ ) 


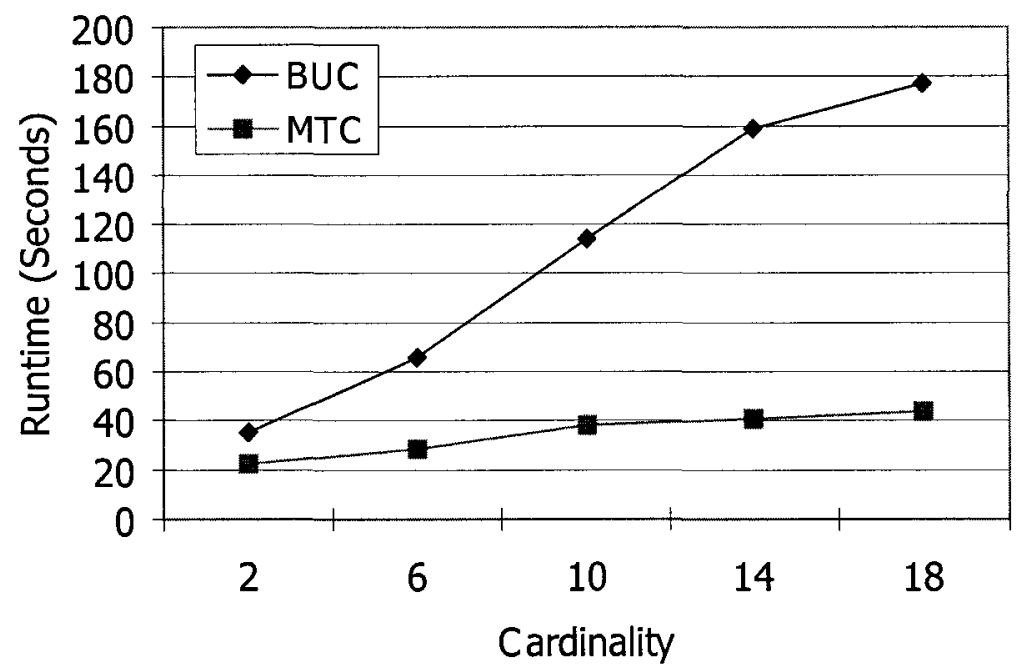

Figure 4.14: Cardinality $($ Table $=$ PSCS, Size $=40,000, \operatorname{MinSup}=10, \mathrm{Zipf}=0)$

\section{Series 4: Dimension (Number of Attributes)}

For the same reason as explained at the beginning of the discussion of the Cardinality series (Series 3), the Student table with seven attributes was also used for the Dimension tests. We performed tests with two different minimum support values, 2000 and 3000. The results, as shown in Figure 4.15 and 4.16, are quite similar. For the two lower numbers of dimensions, 3 and 4, the performance of BUC and MTC was about the same. When the number of dimensions was increased, the runtimes of the two algorithms also increased due to more distinct partitions being processed. In addition, MTC consistently performed the same as or better than BUC. In cases where it performed better, we hypothesize that it did so because of its superior pruning and its ability to perform shared computation.

The data generator mentioned in Section 4.1 cannot create a table with more than four attributes so no tests were done for this series with PSCS tables. 


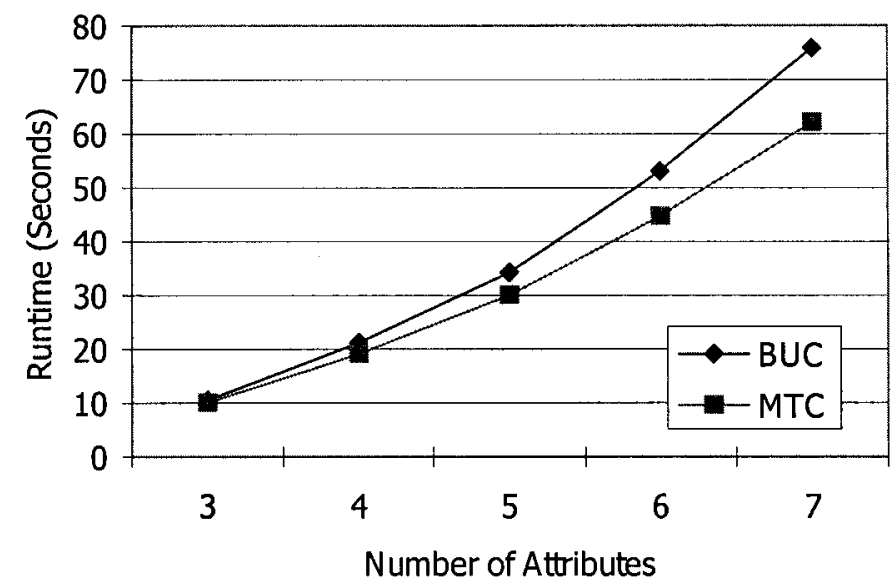

Figure 4.15: Dimension $($ Table $=$ Student, $\operatorname{Size}=30,000, \operatorname{MinSup}=2,000)$

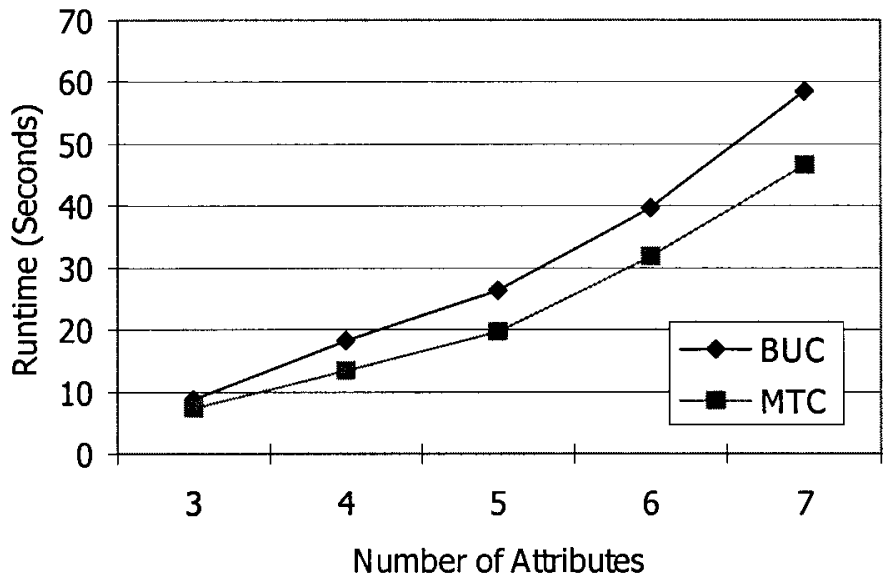

Figure 4.16: Dimension $($ Table $=$ Student, $\operatorname{Size}=30,000, \operatorname{MinSup}=3,000)$ 


\section{Series 5: Data Skew}

The minimum support was set to 100 for the two tests related to skewness. The PSCS table for Figure 4.17 had 20,000 tuples and the one for Figure 4.18 had 40,000 tuples. The results from both charts show the same trend: skewed data made both algorithms performed better and MTC consistently performed better than BUC. Both algorithms use Apriori-like pruning, so they saved time on skewed data by reducing the number of partitions being processed. Again, the more accurate pruning and shared computation of MTC contributed to its better performance. When the Zipf value was decreased, BUC had to read and process more distinct partitions, so the I/O time increased as well as the computation time. But for MTC, the I/O time did not change and the computation time only slightly increased due to wider trees. As a result, the biggest difference in runtimes shown in Figure 4.17 and 4.18 occurred when the Zipf value was 0.

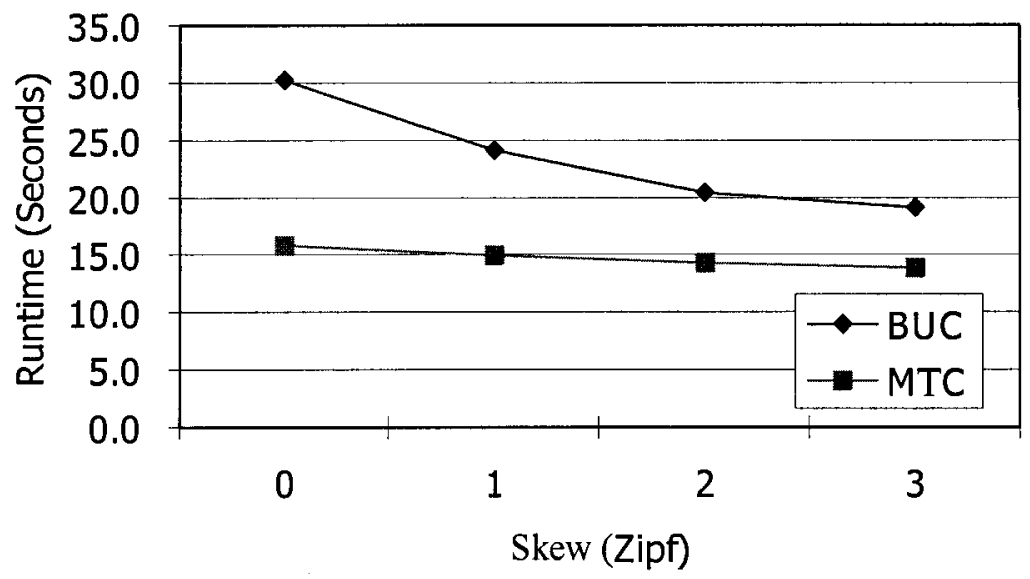

Figure 4.17: Data Skew $($ Table $=$ PSCS, Size $=20,000$, MinSup $=100)$ 


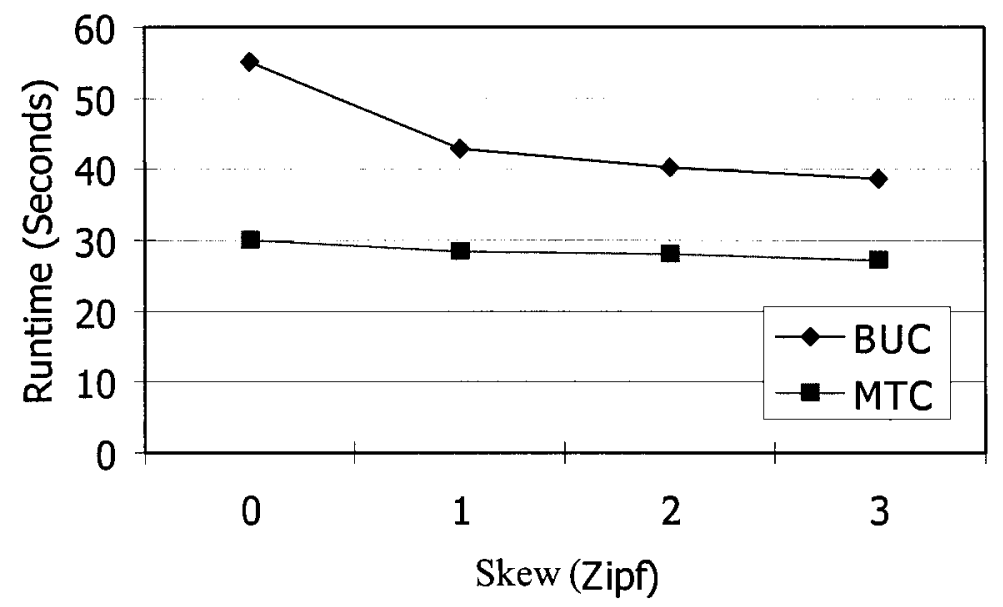

Figure 4.18: Data Skew $($ Table $=$ PSCS, Size $=40,000$, MinSup $=100)$

\subsection{Summary of Findings}

In test series 1, where table size was varied, both BUC and MTC ran longer when the table size increased and their performances were similar. Test series 2, where the minimum support threshold was varied, showed that the two algorithms ran faster as the threshold increased and MTC consistently outperformed BUC. In test series 3, where cardinality was varied, the runtime of BUC increased faster than that of MTC when the cardinality of one attribute increased. Also, MTC outperformed BUC for all tests in series 3. In test series 4, where dimension was varied, both BUC and MTC ran slower when the number of attributes increased and MTC consistently performed better than BUC. In test series 5, where the degree of skewness was varied, the two algorithms improved when the Zipf value increased. In addition, MTC consistently outperformed BUC, and the difference in their runtimes was the greatest when the Zipf value was 0 . 


\section{CHAPTER 5}

\section{CONCLUSIONS}

Section 5.1 provides a brief summary of this thesis and draws the conclusion. Section 5.2 describes several possible areas for future research.

\subsection{Summary}

In this thesis, we gave an overview of on-line analytical processing (OLAP), data cubes and the iceberg cubes. Data cubes can be pre-computed to improve OLAP performance. Since it is not feasible to compute the full data cube when the number of dimensions is high, the iceberg cube problem was introduced.

The iceberg cube problem is to compute all of the partitions with an aggregate value above a user specified minimum support threshold. By identifying and avoiding the computation of the partitions that do not satisfy the minimum support requirement, the performance has been greatly improved. The bottom-up approach, the top-down approach and the integrated top-down and bottom-up approach have been developed by previous studies in this area.

Four algorithms, namely Top-Down Computation, the Multi-Way algorithm, the Bottom-Up Computation, and the Star Cubing algorithm, were reviewed. The main advantage of the top-down approach, as used in Top-Down Computation and the MultiWay Array algorithm, is the shared computation and the main advantage of the bottomup approach, as used in Bottom-Up Computation, is the Apriori-like pruning. The Star 
Cubing algorithm is an integrated top-down and bottom-up approach so it processes both advantages. However, the Star Cubing algorithm stores the whole input table and intermediate results, which makes it a memory intensive algorithm.

The main contribution of the thesis is that we designed, implemented, and tested a new algorithm, called the Multi-Tree Cubing algorithm, for the iceberg cube problem. Like Star Cubing, the Multi-Tree Cubing algorithm integrates top-down and bottom-up approaches, so it benefits from both of their advantages. In addition, the Multi-Tree Cubing algorithm has a more thorough pruning technique than $\mathrm{BUC}$, so that it can further reduce the number of partitions processed. Furthermore, this algorithm processes multiple orderings simultaneously, while TDC only processes one at a time. So the Multi-Tree Cubing algorithm can compute more partitions simultaneously than TDC does and thus can require fewer scans of the input table.

With Apriori-like pruning and shared computation working hand in hand, the Multi-Tree Cubing algorithm performed the same as or better than the BUC algorithm in all five series of experiments we conducted. The varied factors for the five series were the table size, the minimum support, the cardinality, the dimension (number of attributes), and the degree of skewness. We used both real and synthetic databases for these experiments. The synthetic data was generated by a data generator, which is another contribution of the thesis. We used it to generate input tables with different sizes, cardinalities, and degrees of skewness. 


\subsection{Suggestions for Future Research}

Although this thesis compared Multi-Tree Cubing with BUC, further comparisons could be done with other algorithms. For example, a variation of the Star Cubing algorithm for the iceberg cube problem could be designed, implemented, and compared with Multi-Tree Cubing. As well, analysis of the statistical significance of the differences between algorithms could be performed.

The implementation of the Multi-Tree Cubing algorithm used for our research can only compute the iceberg cube using the Count as the aggregate measure. Future research could make the necessary changes so that it could handle other measures. For example, Sum, Minimum, and Maximum could be added in a straightforward manner. Adding the Top-k Average, UB-Average, and Average would be a more challenging goal.

The data generator developed for this research can only create new tables with a fixed structure. In the future, the data generator could be changed to generate data tables with more widely varying formats, including larger numbers of attributes. No technical or theoretical issues that prevented the data generator from handling more than four attributes, so the change should be straightforward. 


\section{References}

[Agrawal and Srikant] R. Agrawal and R. Srikant. Fast algorithms for mining association rules. Proceedings of the 20th International Conference on Very Large Databases. Santiago, Chile, 1994, 487-499.

[Berry and Linoff 1997] M. J. A. Berry and G. Linoff. Data Mining Techniques For Marketing, Sales, and Customer Support. Wiley, 1997.

[Beyer and Ramakrishnan] K. Beyer and R. Ramakrishnan. Bottom-up computation of sparse and iceberg cubes. In Proceedings of the 1999 ACM SIGMOD International Conference on Management of Data, Philadelphia, 1999, 359-370.

[Chou and Zhang 2003] P. L. Chou and X. Zhang, Efficiently computing the top N averages in iceberg cubes, Proceedings of the Twenty-Sixth Australasian Computer Science Conference (ACSC2003), Adelaide, Australia, 2003, 101-109.

[Codd 1993] E. F. Codd, Providing OLAP (On-line Analytical Processing) to UserAnalysts: An IT Mandate, E.F. Codd and Associates, 1993.

[Creeth and Pendse 1995] N. Pendse and R. Creeth. The OLAP Report, 1998. The online report is available on the web at http://www.olapreport.com/Analyses.htm/.

[Endress 2001] B. Endress. “Oracle 9i OLAP RELEASE 2: A Relational Multidimensional Database for Business Intelligence", Oracle Corporation, April 2001.

[Fang et al. 1998] M. Fang, N. Shivakumar, H. Garcia-Molina, R. Motwani, and J. D. Ullman. Computing iceberg queries efficiently. In Proceedings of the 24th International Conference on Very Large Databases (VLDB'98), New York, August 1998, 299-310. 
[Fayyad et al. 1996] U. M. Fayyad, G. Piatetsky-Shapiro, P. Smyth, and R. Uthurusamy, (eds.), Advances in Knowledge Discovery and Data Mining, AAAI Press / The MIT Press, 1996.

[Gray et al. 2001] J. Gray, S. Chaudhuri, A. Bosworth, A. Layman, D. Reichart, M. Venkatrao, F. Pellow and H. Pirahesh. Data cube: A relational aggregation operator generalizing group-by, cross-tab, and sub-totals. Data Mining and Knowledge Discovery, Vol. 1, 1997, 29-54.

[Hamilton and Findlater 2003] L. Findlater and H. J. Hamilton. Iceberg-cube algorithms: An empirical evaluation on synthetic and real data, Intelligent Data Analysis, Vol. 7, No. 2, 2003, 77-97.

[Han et al. 2001] J. Han, J. Pei, G. Dong, and K. Wang. Efficient computation of iceberg cubes with complex measures. In Proceedings of the 2001 ACM SIGMOD International Conference on Management of Data, Santa Barbara, California, 2001, $1-12$.

[Oracle $9 i$ BI 2002] Oracle Corporation. Oracle 9i Business Intelligence Beans, White Paper, Oracle Corporation, September 2002.

[Poosala 1995] V. Poosala. Zipf's Law. Preprint, Computer Science, University of Wisconsin, USA, 1995.

[Xin et al. 2003] D. Xin, J. Han, X. Li, and B. W. Wah. Star-Cubing: Computing iceberg cubes by top-down and bottom-up integration. In Proceedings of the 26th International Conference on Very Large Databases (VLDB'03), Berlin, Germany, 2003. 
[Zhao et al. 1997] Y. Zhao, P. Deshpande, and J. F. Naughton. An array-based algorithm for simultaneous multidimensional aggregates. In Proceedings of the $1997 \mathrm{ACM}$ SIGMOD International Conference on Management of Data, Tucson, Arizona, 1997, 159-170. 


\section{Appendix A}

\section{User's Manual for Multi-Tree Cubing (MTC) and Bottom-Up Computation (BUC) Programs}

The Multi-Tree Cubing (MTC) is an algorithm that computes the iceberg cube with a user specified minimum support. The MTC application was coded in Java and it accesses the MS Access database tables via the ODBC. To run the Multi-Tree Cubing, the Java Runtime environment and MS Access need to be installed and a System DSN mtc needs to be added and mapped to the source database in ODBC.

Go to DOS prompt and then type in java mainMtc to start the MTC application. The main menu has three options. In Option 1 - select table and fields, users can enter the source table name and select the fields for the iceberg cube computation. With Option 2 Set MinSup, users can set a minimum support by entering its percentage of the full table or the actual number of tuples. If a MinSup is not set, then it is set to 1 by default, which means a full data cube computation. Option 3 - Compute Iceberg Cube, will compute the iceberg cube and output the results to a table called MtcResults.

The implementation of Bottom-Up Computation (BUC) is very similar to that of MTC. So we only point out their difference as following:

- BUC needs a User DSN buc to be added in ODBC.

- Go to DOS prompt and then type in Java mainBuc to start the BUC application.

- BUC output the results for the iceberg cube to a table called BucResults. 